\title{
SET THEORY AND OPERATOR ALGEBRAS
}

\author{
ILIJAS FARAH AND ERIC WOFSEY
}

These notes are based on the six-hour Appalachian Set Theory workshop given by Ilijas Farah on February 9th, 2008 at Carnegie Mellon University. The first half of the workshop (Sections 1-4) consisted of a review of Hilbert space theory and an introduction to $\mathrm{C}^{*}$-algebras, and the second half (Sections 5-7) outlined a few set-theoretic problems relating to $\mathrm{C}^{*}$-algebras. The number and variety of topics covered in the workshop was unfortunately limited by the available time.

Good general references on Hilbert spaces and $\mathrm{C}^{*}$-algebras include [8], [12], [16], [45], [55], and [61]. An introduction to spectral theory is given in [9]. Most of the omitted proofs can be found in most of these references. For a survey of applications of set theory to operator algebras, see [58].

Acknowledgments. We owe greatest thanks to Ernest Schimmerling, without whose enthusiasm and support this paper would not have existed. We would like to thank Nik Weaver for giving us a kind permission to include some of his unpublished results. I.F. would like to thank George Elliott, N. Christopher Phillips, Efren Ruiz, Juris Steprāns, Nik Weaver and the second author for many conversations related to the topics presented here. He would also like to thank Sam Coskey, Paul Ellis, Saeed Ghasemi, and Ernest Schimmerling for a number of remarks about the early version of the present paper, and to the anonymous referee for one of the longest and most painstaking reports in the history of mathematics. Special thanks to Gala for her indispensable help with the bibliography.

The early draft of these notes was produced for a series of lectures that the first author gave at the Kobe University in November 2006. He would like to thank Jörg Brendle for organizing this visit and for his hospitality. Some of the results included here were obtained during the first author's stay at the IHES in July 2008. I.F. is partially supported by NSERC.

\section{INTRODUCTION}

A more accurate title for the present paper would be 'Set theory and $\mathrm{C}^{*}$-algebras' but this title was already taken by the excellent Weaver's survey ([58]). Apart from $\mathrm{C}^{*}$-algebras, set theory (both combinatorial and descriptive) has strong connections to the theory of von Neumann algebras,

Date: May 7, 2010. 
and in particular to $\mathrm{II}_{1}$ factors (but see $\S 1.2$ and $\S 1.3$ below). This subject will not be touched upon in the present paper. A very intuitive and approachable introduction to von Neumann algebras can be found in [37].

The fact that there were very few interactions between set theory and theory of operator algebras may be somewhat surprising, not only because John von Neumann played a role in the development of both subjects. Apart from the work of Joel Anderson in the 1970's and some attempts at developing 'noncommutative' set theory, there was virtually no interaction between the two areas until recently.

This situation has dramatically changed in the last decade to the extent that we will not even be able to outline the entire subject in the present paper. Let us instead outline what is covered here. A set-theorist-friendly introduction to operators on Hilbert spaces, continuous function calculus, $\mathrm{C}^{*}$-algebras, and their representation theory is given in sections $\S 1-4$. In $\S 5$ we consider the Calkin algebra as a quantized version of $\mathcal{P}(\mathbb{N}) /$ Fin and consider some problems about the former which are direct translations of theorems (and problems) about the latter. In $\S 6$ we return to the representation theory of $\mathrm{C}^{*}$-algebras and consider two of the most interesting recent applications of set theory to $\mathrm{C}^{*}$-algebras, both due to Akemann and Weaver. These are the construction of a counterexample to Naimark's problem using Jensen's $\diamond$ and a construction of a pure state on $\mathcal{B}(H)$ that is not diagonalizable, using Continuum Hypothesis. In the latter case we present an unpublished result of the first author and Weaver, showing that a substantial weakening of the Continuum Hypothesis suffices. It is not known whether either of these two results can be proved from ZFC alone. In $\S 7$ we give a very brief outline of the solution to the problem whether Calkin algebra has an outer automorphism, due to Phillips-Weaver and the first author.

Applications of set theory to the theory of operator algebras fall into several categories, and we shall now describe (a part of) what is being omitted.

1.1. Nonseparable $\mathbf{C}^{*}$-algebras. Some long-standing open problems in theory of $\mathrm{C}^{*}$-algebras were recently solved in $\mathrm{ZFC}$, by using rather elementary set theory to construct nonseparable $\mathrm{C}^{*}$-algebras with properties not present in separable $\mathrm{C}^{*}$-algebras. We should mention Weaver's construction of a prime $C^{*}$-algebra that is not primitive ([57], see also [17] and [39] for simpler constructions). In [28] and [25] it was demonstrated that even direct limits of full matrix $\mathrm{C}^{*}$-algebras (the nonseparable analogues of UHF algebras, see §3.4.2) can have rather exotic properties. Curiously, all of these results answer long-standing open problems posed by Jacques Dixmier.

1.2. Ultrapowers. Ultrapowers are an indispensable tool both in model theory and in operator algebras, yet until [30] the two theories were developed essentially independently. This can be contrasted to the fact that ultraproducts of Banach spaces were well-studied by logicians. Largely motivated by some questions of Eberhard Kirchberg, a few papers appeared 
recently showing that the structure of ultrapowers and relative commutants of $\mathrm{C}^{*}$-algebras and $\mathrm{II}_{1}$ factors can depend on the choice of the ultrafilter ([29], [24], [26]). A model-theoretic logic suitable for study of $\mathrm{C}^{*}$-algebras and $\mathrm{II}_{1}$ factors, adapted from [10], was developed in [27].

1.3. Classification and descriptive set theory. While the present survey is exclusively concerned with applications of combinatorial set theory, some of the most exciting interactions between operator algebras and set theory were purely descriptive. The abstract classification theory was recently applied to prove determine lower bounds of classification problems for von Neumann algebras, of spectra of $\mathrm{C}^{*}$-algebras. Also, Popa superrigidity developed in the context of $\mathrm{II}_{1}$ factors was indispensable in proving some of the most interesting recent results on countable Borel equivalence relations. But this is an another story.

\section{Hilbert SPACES AND OPERATORS}

We begin with a review of the basic properties of operators on a Hilbert space. Throughout we let $H$ denote a complex infinite-dimensional separable Hilbert space, and we let $\left(e_{n}\right)$ be an orthonormal basis for $H$ (see Example 2.1). For $\xi, \eta \in H$, we denote their inner product by $(\xi \mid \eta)$. We recall that

$$
(\eta \mid \xi)=\overline{(\xi \mid \eta)}
$$

and the norm defined by

$$
\|\xi\|=\sqrt{(\xi \mid \xi)} .
$$

The Cauchy-Schwartz inequality says that

$$
|(\xi \mid \eta)| \leq\|\xi\|\|\eta\| \text {. }
$$

Example 2.1. The space

$$
\ell^{2}(\mathbb{N})=\left\{\left(\alpha_{k}\right)_{k \in \mathbb{N}}: \alpha_{k} \in \mathbb{C},\|\alpha\|^{2}=\sum\left|\alpha_{k}\right|^{2}<\infty\right\}
$$

(sometimes denoted simply by $\ell^{2}$ ) is a Hilbert space under the inner product $(\alpha \mid \beta)=\sum \alpha_{k} \overline{\beta_{k}}$. If we define $e^{n} \in \ell^{2}(\mathbb{N})$ by $e_{k}^{n}=\delta_{n k}$ (the Kronecker's $\delta$ ), then $\left(e^{n}\right)$ is an orthonormal basis for $\ell^{2}$. For any $\alpha \in \ell^{2}, \alpha=\sum \alpha_{n} e^{n}$.

Any Hilbert space has an orthonormal basis, and this can be used to prove that all separable infinite-dimensional Hilbert spaces are isomorphic. Moreover, any two infinite-dimensional Hilbert spaces with the same character density (the minimal cardinality of a dense subset) are isomorphic.

Example 2.2. If $(X, \mu)$ is a measure space,

$$
L^{2}(X, \mu)=\left\{f: X \rightarrow \mathbb{C} \text { measurable }: \int|f|^{2} d \mu<\infty\right\} /\{f: f=0 \text { a.e. }\}
$$

is a Hilbert space under the inner product $(f \mid g)=\int f \bar{g} d \mu$ and with the norm defined by $\|f\|^{2}=\int|f|^{2} d \mu$. 
We will let $a, b, \ldots$ denote linear operators $H \rightarrow H$. We recall that

$$
\|a\|=\sup \{\|a \xi\|: \xi \in H,\|\xi\|=1\} .
$$

If $\|a\|<\infty$, we say $a$ is bounded. An operator is bounded if and only if it is continuous. We denote the algebra of all bounded operators on $H$ by $\mathcal{B}(H)$ (some authors use $L(H)$ ), and throughout the paper all of our operators will be bounded. We define the adjoint $a^{*}$ of $a$ to be the unique operator satisfying

$$
(a \xi \mid \eta)=\left(\xi \mid a^{*} \eta\right)
$$

for all $\xi, \eta \in H$. Note that since an element of $H$ is determined by its inner products with all other elements of $H$ (e.g., take an orthonormal basis), an operator $a$ is determined by the values of $(a \xi \mid \eta)$ for all $\xi, \eta$ or even by the values $\left(e e_{m} \mid e_{n}\right)$ for $m$ and $n$ in $\mathbb{N}$.

Lemma 2.3. For all $a, b$ we have

(1) $\left(a^{*}\right)^{*}=a$

(2) $(a b)^{*}=b^{*} a^{*}$

(3) $\|a\|=\left\|a^{*}\right\|$

(4) $\|a b\| \leq\|a\| \cdot\|b\|$

(5) $\left\|a^{*} a\right\|=\|a\|^{2}$

Proof. These are all easy calculations. For example, for (5), for $\|\xi\|=1$,

$$
\|a \xi\|^{2}=(a \xi \mid a \xi)=\left(\xi \mid a^{*} a \xi\right) \leq\|\xi\| \cdot\left\|a^{*} a \xi\right\| \leq\left\|a^{*} a\right\|,
$$

the first inequality holding by Cauchy-Schwartz. Taking the sup over all $\xi$, we obtain $\|a\|^{2} \leq\left\|a^{*} a\right\|$. Conversely,

$$
\left\|a^{*} a\right\| \leq\left\|a^{*}\right\|\|a\|=\|a\|^{2}
$$

by (3) and (4).

The first four parts of this say that $\mathcal{B}(H)$ is a Banach *algebra (or a Banach algebra with involution ${ }^{*}$ ) and (5) (sometimes called the $C^{*}$-equality) says that $\mathcal{B}(H)$ is a $\mathrm{C}^{*}$-algebra.

Definition 2.4. An (abstract) $C^{*}$-algebra is a Banach *-algebra satisfying the $\mathrm{C}^{*}$-equality, $\left\|a^{*} a\right\|=\|a\|^{2}$ for all $a$.

2.1. Normal operators and the spectral theorem. In this section we introduce some distinguished classes of operators in $\mathcal{B}(H)$, such as normal and self-adjoint operators (cf. §3.0.1).

Example 2.5. Assume $(X, \mu)$ is a probability measure space. If $H_{0}=$ $L^{2}(X, \mu)$ and $f: X \rightarrow \mathbb{C}$ is bounded and measurable, then

$$
H_{0} \ni g \stackrel{m_{f}}{\longmapsto} f g \in H_{0}
$$

is a bounded linear operator. We have $\left\|m_{f}\right\|=\|f\|_{\infty}$ and

$$
m_{f}^{*}=m_{\bar{f}} \text {. }
$$


Hence $m_{f}^{*} m_{f}=m_{f} m_{f}^{*}=m_{|f|^{2}}$. We call operators of this form multiplication operators.

An operator $a$ is normal if $a a^{*}=a^{*} a$. Clearly, all multiplication operators are normal. Normal operators have a nice structure theory, which is summarized in the following theorem.

Theorem 2.6 (Spectral Theorem). If a is a normal operator then there is a probability measure space $(X, \mu)$, a measurable function $f$ on $X$, and a Hilbert space isomorphism $\Phi: L^{2}(X, \mu) \rightarrow H$ such that $\Phi a \Phi^{-1}=m_{f}$.

Proof. For an elegant proof using Corollary 3.13 see [9, Theorem 2.4.5].

That is, every normal operator is a multiplication operator for some identification of $H$ with an $L^{2}$ space. Conversely, every multiplication operator is clearly normal. If $X$ is discrete and $\mu$ is counting measure, the characteristic functions of the points of $X$ form an orthonormal basis for $L^{2}(X, \mu)$ and the spectral theorem says that $a$ is diagonalized by this basis. In general, the spectral theorem says that normal operators are "measurably diagonalizable".

If $\Phi: H_{1} \rightarrow H_{2}$ is an isomorphism between Hilbert spaces, then

$$
a \mapsto \operatorname{Ad} \Phi(a)=\Phi a \Phi^{-1}
$$

is an isomorphism between $\mathcal{B}\left(H_{1}\right)$ and $\mathcal{B}\left(H_{2}\right)$. The operator $\operatorname{Ad} \Phi(a)$ is just $a$ with its domain and range identified with $H_{2}$ via $\Phi$.

Our stating of the Spectral Theorem is rather premature in the formal sense since we are going to introduce some of the key notions used in its proof later on, in $\S 2.2$ and $\S 3.2$. This was motivated by the insight that the Spectral Theorem provides to theory of $\mathrm{C}^{*}$-algebras.

An operator $a$ is self-adjoint if $a=a^{*}$. Self-adjoint operators are obviously normal. For any $b \in \mathcal{B}(H)$, the "real" and "imaginary" parts of $b$, defined by $b_{0}=\left(b+b^{*}\right) / 2$ and $b_{1}=\left(b-b^{*}\right) / 2 i$, are self-adjoint and satisfy $b=b_{0}+i b_{1}$. Thus any operator is a linear combination of self-adjoint operators. It is easy to check that an operator is normal if and only if its real and imaginary parts commute, so the normal operators are exactly the linear combinations of commuting self-adjoint operators.

Example 2.7. The real and imaginary parts of a multiplication operator $m_{f}$ are $m_{\operatorname{Re} f}$ and $m_{\operatorname{Im} f}$. A multiplication operator $m_{f}$ is self-adjoint if and only if $f$ is real (a.e.). By the spectral theorem, all self-adjoint operators are of this form.

It is easy to verify that for any $a \in \mathcal{B}(H)$ and $\xi, \eta \in H$ the following so-called polarization identity holds

$$
(a \xi \mid \eta)=\frac{1}{4} \sum_{k=0}^{3} i^{k}\left(a\left(\xi+i^{k} \eta\right) \mid \xi+i^{k} \eta\right) .
$$


Proposition 2.8. An operator $a$ is self-adjoint if and only if $(a \xi \mid \xi)$ is real for all $\xi$.

Proof. First, note that

$$
\left(\left(a-a^{*}\right) \xi \mid \xi\right)=(a \xi \mid \xi)-\left(a^{*} \xi \mid \xi\right)=(a \xi \mid \xi)-(\xi \mid a \xi)=(a \xi \mid \xi)-\overline{(a \xi \mid \xi)} .
$$

Thus $(a \xi \mid \xi)$ is real for all $\xi$ if and only if $\left(\left(a-a^{*}\right) \xi \mid \xi\right)=0$ for all $\xi$. But by polarization, the operator $a-a^{*}$ is entirely determined by the values $\left(\left(a-a^{*}\right) \xi \mid \xi\right)$, so this is equivalent to $a-a^{*}=0$.

A self-adjoint operator $b$ is called positive if $(b \xi \mid \xi) \geq 0$ for all $\xi \in H$. In this situation we write $b \geq 0$. By Proposition 2.8, positive operators are self-adjoint. For instance, a multiplication operator $m_{f}$ is positive if and only if $f \geq 0$ (a.e.). By the spectral theorem, all positive operators are of the form $m_{f}$ for $f \geq 0$ a.e.

Exercise 2.9. For any self-adjoint $a \in \mathcal{B}(H)$ we can write $a=a_{0}-a_{1}$ for some positive operators $a_{0}$ and $a_{1}$. (Hint: Use the spectral theorem.)

Proposition 2.10. An operator $b$ is positive if and only if $b=a^{*} a$ for some (non-unique) a. This a may be chosen to be positive.

Proof. For the converse implication note that $\left(a^{*} a \xi \mid \xi\right)=(a \xi \mid a \xi)=\|a \xi\|^{2} \geq$ 0 . For the direct implication, assume $b$ is positive. By the spectral theorem we may assume $b=m_{f}$ for $f \geq 0$. Let $a=m_{\sqrt{f}}$.

We say that $p \in \mathcal{B}(H)$ is a projection if $p^{2}=p^{*}=p$.

Lemma 2.11. $p$ is a projection if and only if it is the orthogonal projection onto a closed subspace of $H$.

Proof. Any linear projection $p$ onto a closed subspace of $H$ satisfies $p=$ $p^{2}$, and orthogonal projections are exactly those that also satisfy $p=p^{*}$. Conversely, suppose $p$ is a projection. Then $p$ is self-adjoint, so we can write $p=m_{f}$ for $f: X \rightarrow \mathbb{C}$, and we have $f=f^{2}=\bar{f}$. Hence $f(x) \in\{0,1\}$ for (almost) all $x$. We then set $A=f^{-1}(\{1\})$, and it is easy to see that $p$ is the orthogonal projection onto the closed subspace $L^{2}(A) \subseteq L^{2}(X)$.

If $E \subseteq H$ is a closed subspace, we denote the projection onto $E$ by $\operatorname{proj}_{E}$.

We denote the identity operator on $H$ by $I$ (some authors use 1). An operator $u$ is unitary if $u u^{*}=u^{*} u=I$. This is equivalent to $u$ being invertible and satisfying

$$
(\xi \mid \eta)=\left(u^{*} u \xi \mid \eta\right)=(u \xi \mid u \eta)
$$

for all $\xi, \eta \in H$. That is, an operator is unitary if and only if it is a Hilbert space automorphism of $H$. Unitary operators are obviously normal. For instance, a multiplication operator $m_{f}$ is unitary if $m_{f}\left(m_{f}\right)^{*}=I$, or equivalently, if $|f|^{2}=1$ (a.e.). By the spectral theorem, all unitaries are of this form. 
An operator $v$ is a partial isometry if

$$
p=v v^{*} \text { and } q=v^{*} v
$$

are both projections. Partial isometries are essentially isomorphisms (isometries) between closed subspaces of $H$ : For every partial isometry $v$ there is a closed subspace $H_{0}$ of $H$ such that $v\left\lceil H_{0}\right.$ is an isometry into a closed subspace of $H$ and $v\left\lceil H_{0}^{\perp} \equiv 0\right.$. (As usually, $H_{0}^{\perp}$ is the orthogonal complement of $H_{0},\left\{\xi:(\xi \mid \eta)=0\right.$ for all $\left.\eta \in H_{0}\right\}$.) However, as the following example shows, partial isometries need not be normal.

Example 2.12. Let $\left(e_{n}\right)$ be an orthonormal basis of $H$. We define the unilateral shift $S$ by $S\left(e_{n}\right)=e_{n+1}$ for all $n$. Then $S^{*}\left(e_{n+1}\right)=e_{n}$ and $S^{*}\left(e_{0}\right)=0$. We have $S^{*} S=I$ but $S S^{*}=\operatorname{proj}_{\overline{\operatorname{span}}\left\{e_{n}\right\}_{n \geq 1}}$.

Any complex number $z$ can be written as $z=r e^{i \theta}$ for $r \geq 0$ and $\left|e^{i \theta}\right|=1$. Considering $\mathbb{C}$ as the set of operators on a one-dimensional Hilbert space, there is an analogue of this on an arbitrary Hilbert space.

Theorem 2.13 (Polar Decomposition). Any $a \in \mathcal{B}(H)$ can be written as $a=b v$ where $b$ is positive and $v$ is a partial isometry.

Proof. See e.g., [45, Theorem 3.2.17 and Remark 3.2.18].

However, this has less value as a structure theorem than than one might think, since $b$ and $v$ may not commute. While positive operators and partial isometries are both fairly easy to understand, polar decomposition does not always make arbitrary operators easy to understand. For example, it is easy to show that positive operators and partial isometries always have nontrivial closed invariant subspaces, but it is a famous open problem whether this is true for all operators.

2.2. The spectrum of an operator. The spectrum of an operator $a$ is

$$
\sigma(a)=\{\lambda \in \mathbb{C}: a-\lambda I \text { is not invertible }\} .
$$

For a finite-dimensional matrix, the spectrum is the set of eigenvalues.

Example 2.14. A multiplication operator $m_{f}$ is invertible if and only if there is some $\epsilon>0$ such that $|f|>\epsilon$ (a.e.). Thus since $m_{f}-\lambda I=m_{f-\lambda}$, $\sigma\left(m_{f}\right)$ is the essential range of $f$ (the set of $\lambda \in \mathbb{C}$ such that for every neighborhood $U$ of $\lambda, f^{-1}(U)$ has positive measure).

Lemma 2.15. If $\|a\|<1$ then $I-a$ is invertible in $\mathcal{B}(H)$.

Proof. The series $b=\sum_{n=0}^{\infty} a^{n}$ is convergent and hence in $\mathcal{B}(H)$. By considering partial sums one sees that $(I-a) b=b(I-a)=I$.

The following Lemma is an immediate consequence of the Spectral Theorem. However, since its part (1) is used in the proof of the latter, we provide its proof.

Lemma 2.16. Let $a \in \mathcal{B}(H)$. 
(1) $\sigma(a)$ is a compact subset of $\mathbb{C}$.

(2) $\sigma\left(a^{*}\right)=\{\bar{\lambda}: \lambda \in \sigma(a)\}$.

(3) If $a$ is normal, then $a$ is self-adjoint if and only if $\sigma(a) \subseteq \mathbb{R}$.

(4) If $a$ is normal, then $a$ is positive if and only if $\sigma(a) \subseteq[0, \infty)$.

Proof of (1). If $|\lambda|>\|a\|$ then $a-\lambda \cdot I=\lambda\left(\frac{1}{\lambda} a-I\right)$ is invertible by Lemma 2.15, and therefore $\sigma(a)$ is bounded.

We shall now show that the set of invertible elements is open. Fix an invertible $a$. Since the multiplication is continuous, we can find $\epsilon>0$ such that for every $b$ in the $\epsilon$-ball centered at $a$ there is $c$ such that both $\| I-$ $b c \|<1$ and $\|I-c b\|<1$. By Lemma 2.15 there are $d_{1}$ and $d_{2}$ such that $b c d_{1}=d_{2} c b=I$. Then we have

$$
c d_{1}=I \cdot c d_{1}=d_{2} c b c d_{1}=d_{2} c \cdot I=d_{2} c
$$

and therefore $c d_{1}=d_{2} c$ is the inverse of $b$.

Let $a$ be an arbitrary operator. If $\lambda \notin \sigma(a)$ then by the above there is an $\epsilon>0$ such that every $b$ in the $\epsilon$-ball centered at $a-\lambda I$ is invertible. In particular, if $\left|\lambda^{\prime}-\lambda\right|<\epsilon$ then $\lambda^{\prime} \notin \sigma(a)$, concluding the proof that $\sigma(a)$ is compact.

\section{3. $\mathrm{C}^{*}$-ALGEBRAS}

We say that a concrete $C^{*}$-algebra is a norm-closed ${ }^{*}$-subalgebra of $\mathcal{B}(H)$. For $X \subseteq \mathcal{B}(H)$ by $C^{*}(X)$ we denote the $C^{*}$-algebra generated by $X$, i.e., the norm-closure of the algebra of all ${ }^{*}$-polynomials in elements of $X$. Equivalently, $C^{*}(X)$ is the intersection of all $\mathrm{C}^{*}$-subalgebras of $\mathcal{B}(H)$ including $X$.

When talking about $\mathrm{C}^{*}$-algebras, everything is 'starred': subalgebras

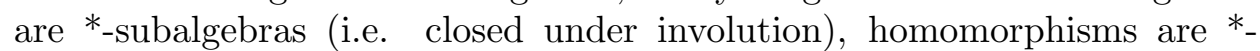
homomorphisms (i.e. preserve the involution), etc.

Definition 3.1. An (abstract) $C^{*}$-algebra is a Banach algebra with involution that satisfies the $\mathrm{C}^{*}$-equality $\left\|a a^{*}\right\|=\|a\|^{2}$ for all $a$. That is, it is a Banach space with a product and involution satisfying Lemma 2.3.

A $\mathrm{C}^{*}$-algebra is unital if it has a unit (multiplicative identity). For unital $\mathrm{C}^{*}$-algebras, we can talk about the spectrum of an element.

Lemma 3.2. Every $C^{*}$-algebra $A$ is contained in a unital $C^{*}$-algebra $\tilde{A} \cong$ $A \oplus \mathbb{C}$.

Proof. On $A \times \mathbb{C}$ define the operations as follows: $(a, \lambda)(b, \xi)=(a b+\lambda b+$ $\xi a, \lambda \xi),(a, \lambda)^{*}=\left(a^{*}, \bar{\lambda}\right)$ and $\|(a, \lambda)\|=\sup _{\|b\| \leq 1}\|a b+\lambda b\|$ and check that this is still a $\mathrm{C}^{*}$-algebra.

A straightforward calculation shows that $(0,1)$ is the unit of $\tilde{A}$ and that $A \ni a \mapsto(a, 0) \in \tilde{A}$ is an isomorphic embedding of $A$ into $\tilde{A}$.

Exercise 3.3. Work out the details of the proof of Lemma 3.2. 
We call $\tilde{A}$ the unitization of $A$. By passing to the unitization, we can talk about the spectrum of an element of a nonunital $\mathrm{C}^{*}$-algebra. The unitization retains many of the properties of the algebra $A$, and many results are proved by first considering the unitization. However, some caution is advised; for example, the unitization is never a simple algebra. (Recall that an algebra is simple if it has no nontrivial (two-sided) ideals. In case of $\mathrm{C}^{*}$-algebras, an algebra is simple if it has no nontrivial closed ideals. Such ideals are automatically self-adjoint.)

If $A$ and $B$ are unital and $A \subseteq B$ we say $A$ is a unital subalgebra of $B$ if the unit of $B$ belongs to $A$ (that is, $B$ has the same unit as $A$ ).

3.0.1. Types of operators in $C^{*}$-algebras. We import all of our terminology for distinguished classes of operators in $\mathcal{B}(H)$ (normal, self-adjoint, projections, etc.) to describe elements of any $\mathrm{C}^{*}$-algebra (cf. $\left.§ 2.1\right)$. More precisely, for an operator $a$ in a $\mathrm{C}^{*}$-algebra $A$ we say that

(1) $a$ is normal if $a a^{*}=a^{*} a$,

(2) $a$ is self-adjoint (or Hermitian) if $a=a^{*}$,

(3) $a$ is a projection if $a^{2}=a^{*}=a$,

(4) $a$ is positive (or $a \geq 0$ ) if $a=b^{*} b$ for some $b$,

(5) If $A$ is unital then $a$ is unitary if $a a^{*}=a^{*} a=I$.

Note that a positive element is automatically self-adjoint. For self-adjoint elements $a$ and $b$ write $a \leq b$ if $b-a$ is positive.

3.1. Some examples of $\mathbf{C}^{*}$-algebras. Let us consider several important $\mathrm{C}^{*}$-algebras and classes of $\mathrm{C}^{*}$-algebras (see also [18]).

3.1.1. $C_{0}(X)$. Let $X$ be a locally compact Hausdorff space. Then

$$
C_{0}(X)=\{f: X \rightarrow \mathbb{C}: f \text { is continuous and vanishes at } \infty\}
$$

is a $\mathrm{C}^{*}$-algebra with the involution $f^{*}=\bar{f}$. Here "vanishes at $\infty$ " means that $f$ extends continuously to the one-point compactification $X \cup\{\infty\}$ of $X$ such that the extension vanishes at $\infty$. Equivalently, for any $\epsilon>0$, there is a compact set $K \subseteq X$ such that $|f(x)|<\epsilon$ for $x \notin K$. In particular, if $X$ itself is compact, all continuous functions vanish at $\infty$, and we write $C_{0}(X)=C(X)$.

$C_{0}(X)$ is abelian, so in particular every element is normal. $C_{0}(X)$ is unital if and only if $X$ is compact (iff the constant function 1 vanishes at $\infty$ ). The unitization of $C_{0}(X)$ is $C\left(X^{*}\right)$, where $X^{*}$ is the one-point compactification of $X$. For $f \in C_{0}(X)$, we have:

$f$ is self-adjoint if and only if $\operatorname{range}(f) \subseteq \mathbb{R}$.

$f$ is positive if and only if range $(f) \subseteq[0, \infty)$.

$f$ is a projection if and only if $f^{2}(x)=f(x)=\overline{f(x)}$

if and only if range $(f) \subseteq\{0,1\}$

if and only if $f=\chi_{U}$ for a clopen $U \subseteq X$.

For any $f \in C_{0}(X), \sigma(f)=\operatorname{range}(f)$. 
3.1.2. Full matrix algebras. $M_{n}(\mathbb{C})$, the set of $n \times n$ complex matrices is a unital $\mathrm{C}^{*}$-algebra. In fact, $M_{n}(\mathbb{C}) \cong \mathcal{B}\left(\mathbb{C}^{n}\right)$, where $\mathbb{C}^{n}$ is the $n$-dimensional complex Hilbert space.

adjoint, unitary: the usual meaning.

self-adjoint: Hermitian.

positive: positive semidefinite.

$\sigma(a)$ : the set of eigenvalues.

The spectral theorem on $M_{n}(\mathbb{C})$ is the spectral theorem of elementary linear algebra: normal matrices are diagonalizable.

3.1.3. $L^{\infty}(X, \mu)$. If $(X, \mu)$ is a measure space, then the space $L^{\infty}(X, \mu)$ of all essentially bounded $\mu$-measurable functions on $X$ can be identified with the space of all multiplication operators (see Example 2.5). Then $L^{\infty}(X, \mu)$ is a concrete $C^{*}$-algebra acting on $L^{2}(X, \mu)$. It is easy to see that $\left\|m_{f}\right\|$ is equal to the essential supremum of $f$,

$$
\|f\|_{\infty}=\sup \{t \geq 0: \mu\{x:|f(x)|>t\}>0\} .
$$

3.1.4. The algebra of compact operators. It is equal to ${ }^{1}$

$$
\begin{aligned}
\mathcal{K}(H) & =C^{*}(\{a \in \mathcal{B}(H): a[H] \text { is finite-dimensional }\}) \\
& =\{a \in \mathcal{B}(H): a[\text { unit ball }] \text { is precompact }\} \\
& =\{a \in \mathcal{B}(H): a[\text { unit ball }] \text { is compact }\} .
\end{aligned}
$$

(Note that $\mathcal{K}(H)$ is denoted by $C(H)$ in [44] and by $\mathbf{B}_{0}(H)$ in [45], by analogy with $C_{0}(X)$.) We write $r_{n}=\operatorname{proj}_{\overline{\operatorname{span}}\left\{e_{j} \mid j \leq n\right\}}$ for a fixed basis $\left\{e_{n}\right\}$ of $H$. Then for $a \in \mathcal{B}(H)$, the following are equivalent:

(1) $a \in \mathcal{K}(H)$,

(2) $\lim _{n}\left\|a\left(I-r_{n}\right)\right\|=0$,

(3) $\lim _{n}\left\|\left(I-r_{n}\right) a\right\|=0$.

Note that if $a$ is self-adjoint then

$$
\left\|a\left(I-r_{n}\right)\right\|=\left\|\left(a\left(I-r_{n}\right)\right)^{*}\right\|=\left\|\left(I-r_{n}\right) a\right\| .
$$

In the following exercises and elsewhere, 'ideal' always stands for a two-sided, closed, self-adjoint ideal. Actually, the las property follows from the previous ones since every two-sided closed ideal in a $\mathrm{C}^{*}$-algebra is automatically selfadjoint.

Exercise 3.4. Prove $\mathcal{K}(H)$ is an ideal of $\mathcal{B}(H)$. That is, prove that for $a \in \mathcal{K}(H)$ and $b \in \mathcal{B}(H)$ both $a b$ and $b a$ belong to $\mathcal{K}(H)$, that $\mathcal{K}(H)$ is norm-closed and that $b \in \mathcal{K}(H)$ if and only if $b^{*} \in \mathcal{K}(H)$.

Exercise 3.5. (1) Prove that $\mathcal{K}(H)$ is the unique ideal of $\mathcal{B}(H)$ when $H$ is a separable Hilbert space.

(2) Assume $\kappa$ is an infinite cardinal. Show that the number of proper ideals of $\mathcal{B}\left(\ell^{2}(\kappa)\right)$ is equal to the number of infinite cardinals $\leq \kappa$.

\footnotetext{
${ }^{1}$ The third equality is a nontrivial fact specific to the Hilbert space; see [45, Theorem 3.3 .3 (iii)]
} 
3.1.5. The Calkin algebra. This is an example of an abstract $\mathrm{C}^{*}$-algebra. The quotient $\mathcal{C}(H)=\mathcal{B}(H) / \mathcal{K}(H)$ is called the Calkin algebra. It is sometimes denoted by $Q$ or $Q(H)$. We write $\pi: \mathcal{B}(H) \rightarrow \mathcal{C}(H)$ for the quotient map. The norm on $\mathcal{C}(H)$ is the usual quotient norm for Banach spaces:

$$
\|\pi(a)\|=\inf \{\|b\|: \pi(b)=\pi(a)\}
$$

The Calkin algebra turns out to be a very "set-theoretic" $\mathrm{C}^{*}$-algebra, analogous to the Boolean algebra $\mathcal{P}(\mathbb{N}) /$ Fin.

We shall give more examples of $\mathrm{C}^{*}$-algebras in $\S 3.4$, after proving a fundamental result.

3.2. Automatic continuity and the Gelfand transform. In this section we prove two important results. First, any *-homomorphism between $\mathrm{C}^{*}$ algebras is norm-decreasing (Lemma 3.9) and second, every unital abelian $\mathrm{C}^{*}$-algebra is of the form $C(X)$ for some compact Hausdorff space $X$ (Theorem 3.10).

Lemma 3.6. If $a$ is normal then $\left\|a^{2^{n}}\right\|=\|a\|^{2^{n}}$ for all $n \in \mathbb{N}$.

Proof. Repeatedly using the $\mathrm{C}^{*}$-equality and normality of $a$ we have

$$
\left\|a^{2}\right\|=\left(\left\|\left(a^{*}\right)^{2} a^{2}\right\|\right)^{1 / 2}=\left(\left\|\left(a^{*} a\right)^{*}\left(a^{*} a\right)\right\|\right)^{1 / 2}=\left\|a^{*} a\right\|=\|a\|^{2} .
$$

The Lemma now follows by a straightforward induction.

Exercise 3.7. Find $a \in \mathcal{B}(H)$ such that $\|a\|=1$ and $a^{2}=0$. (Hint: Choose $a$ to be a partial isometry.)

It can be proved that a $\mathrm{C}^{*}$-algebra is abelian if and only if it contains no nonzero element $a$ such that $a^{2}=0$ (see [12, II.6.4.14]).

The spectral radius of an element $a$ of a $\mathrm{C}^{*}$-algebra is defined as

$$
r(a)=\max \{|\lambda|: \lambda \in \sigma(a)\} .
$$

Lemma 3.8. Let $A$ be $a C^{*}$-algebra and $a \in A$ be normal. Then $\|a\|=r(a)$.

Sketch of a proof. It can be proved (see [9, Theorem 1.7.3], also the first line of the proof of Lemma 2.16) that for an arbitrary $a$ we have

$$
\lim _{n}\left\|a^{n}\right\|^{1 / n}=r(a),
$$

in particular, the limit on the left hand side exists. By Lemma 3.6, for a normal $a$ this limit is equal to $\|a\|$.

Lemma 3.9. Any *-homomorphism $\Phi: A \rightarrow B$ between $C^{*}$-algebras is a contraction (in particular, it is continuous). Therefore, any (algebraic) isomorphism between $C^{*}$-algebras is an isometry.

Proof. By passing to the unitizations, we may assume $A$ and $B$ are unital and $\Phi$ is unital as well (i.e., $\Phi\left(I_{A}\right)=I_{B}$ ). 
Note that for any $a \in A, \sigma(\Phi(a)) \subseteq \sigma(a)$ (by the definition of the spectrum). Thus for $a$ normal, using Lemma 3.8,

$$
\begin{aligned}
\|a\| & =\sup \{|\lambda|: \lambda \in \sigma(a)\} \\
& \geq \sup \{|\lambda|: \lambda \in \sigma(\Phi(a))\} \\
& =\|\Phi(a)\| .
\end{aligned}
$$

For general $a, a a^{*}$ is normal so by the $\mathrm{C}^{*}$-equality we have

$$
\|a\|=\sqrt{\left\|a a^{*}\right\|} \geq \sqrt{\left\|\Phi\left(a a^{*}\right)\right\|}=\|\Phi(a)\| .
$$

The reader may want to compare the last sentence of Lemma 3.9 with the situation in Banach space theory, where isomorphism and isometry drastically differ - even in the case of the Hilbert space (see [43]).

For a unital abelian $\mathrm{C}^{*}$-algebra $A$ consider its spectrum

$$
\hat{A}=\{\phi: A \rightarrow \mathbb{C}: \phi \text { is a nonzero algebra homomorphism }\} .
$$

It is not difficult to see that every homomorphism from $A$ into $\mathbb{C}$ is a ${ }_{\text {- }}$ homomorphism. By Lemma 3.9 each $\phi \in \hat{A}$ is a contraction. Also $\phi(I)=1$, and therefore $\hat{A}$ is a subset of the unit ball of the Banach space dual $A^{*}$ of $A$. Since it is obviously closed, it is weak*-compact by the Banach-Alaoglu theorem.

Theorem 3.10 (Gelfand-Naimark). If $A$ is unital and abelian $C^{*}$-algebra and $\hat{A}$ is its spectrum, then $A \cong C(\hat{A})$.

Proof. For $a \in A$ the map $f_{a}: \hat{A} \rightarrow \mathbb{C}$ defined by

$$
f_{a}(\phi)=\phi(a)
$$

is continuous in the weak*-topology. The transformation

$$
A \ni a \longmapsto f_{a} \in C(X)
$$

is the Gelfand transform of $a$. An easy computation shows that the Gelfand transform is a ${ }^{*}$-homomorphism, and therefore by Lemma 3.9 continuous. We need to show it is an isometry.

For $b \in A$ we claim that $b$ is not invertible if and only if $\phi(b)=0$ for some $\phi \in \hat{A}$. Only the forward direction requires a proof. Fix a noninvertible $b$. The $J_{b}=\{x b: x \in A\}$ is a proper (two-sided since $A$ is abelian) ideal containing $b$. Let $J \supseteq J_{b}$ be a maximal proper two sided (not necessarily closed and not necessarily self-adjoint) ideal. Lemma 2.15 implies that $\|I-b\| \geq 1$ for all $b \in J$. Hence the closure of $J$ is still proper, and by maximality $J$ is a closed ideal. Every closed two-sided ideal in a $\mathrm{C}^{*}$ algebra is automatically self-adjoint (see [8, p.11]). Therefore the quotient map $\phi_{J}$ from $A$ to $A / J$ is a ${ }^{*}$-homomorphism. Since $A$ is abelian, by the maximality of $J$ the algebra $A / J$ is a field. For any $a \in A / J$, Lemma 3.8 implies that $\sigma(a)$ is nonempty, and for any $\lambda \in \sigma(a), a-\lambda I=0$ since $A / J$ 
is a field. Thus $A / J$ is generated by $I$ and therefore isomorphic to $\mathbb{C}$, so $\phi_{J} \in X$. Clearly $\phi_{J}(b)=0$.

Therefore range $\left(f_{a}\right)=\sigma(a)$ for all $a$. Lemma 3.8 implies

$$
\|a\|=\max \{|\lambda|: \lambda \in \sigma(a)\}=\left\|f_{a}\right\| .
$$

Thus the algebra $B=\left\{f_{a}: a \in A\right\}$ is isometric to $A$. Since it separates the points in $X$, by the Stone-Weierstrass theorem (e.g., [45, Theorem 4.3.4]) $B$ is norm-dense in $C(X)$. Being isometric to $A$, it is closed and therefore equal to $C(X)$.

The following exercise shows that the category of abelian unital $\mathrm{C}^{*}$ algebras is contravariantly equivalent to the category of compact Hausdorff spaces.

Exercise 3.11. Assume $X$ and $Y$ are compact Hausdorff spaces and $\Phi: C(X) \rightarrow$ $C(Y)$ is a ${ }^{*}$-homomorphism.

(1) Prove that there exists a unique continuous $f: Y \rightarrow X$ such that $\Phi(a)=a \circ f$ for all $a \in C(X)$.

(2) Prove that $\Phi$ is a surjection if and only if $f$ is an injection.

(3) Prove that $\Phi$ is an injection if and only if $f$ is a surjection.

(4) Prove that for every $f: Y \rightarrow X$ there exists a unique $\Phi: C(X) \rightarrow$ $C(Y)$ such that (1)-(3) above hold.

The following exercise provides an alternative construction of a ČechStone compactification $\beta X$ of a completely regular space $X$. (Recall that a topological space is completely regular if it is homeomorphic to a subspace of some Hilbert cube, $[0,1]^{J}$.)

Exercise 3.12. Let $X$ be a completely regular space and let $A$ be the *-algebra $C_{b}(X, \mathcal{B})$ of all continuous bounded functions from $X$ into $\mathbb{C}$, equipped with the sup norm.

(1) Prove that $A$ is a $\mathrm{C}^{*}$-algebra.

(2) Let $\gamma X$ denote the compact Hausdorff space such that $C(\gamma X)$ is isomorphic to $C_{b}(X, \mathcal{B})$. Show that there is a homeomorphic embed$\operatorname{ding} f: X \rightarrow \gamma X$ such that $\Phi(a)=a \circ f$ represents the isomorphism of $C(\gamma X)$ and $C_{b}(X, \mathcal{B})$.

(3) Prove that $f[X]$ is dense in $\gamma X$.

(4) Prove that every continuous real-valued function on $f[X]$ has unique continuous extension with domain $\gamma X$.

3.3. Continuous functional calculus. Recall that $\sigma(a)$ is always a compact subset of $\mathbb{C}$ (Lemma 2.16). Theorem 2.6 (Spectral Theorem) is a consequence of the following Corollary and some standard manipulations; see [9, Theorem 2.4.5].

Corollary 3.13. If $a \in \mathcal{B}(H)$ is normal then $C^{*}(a, I) \cong C(\sigma(a))$. 
Proof. We first prove that $C^{*}(a, I)$ is isomorphic to $C\left(\sigma_{0}(a)\right)$, where $\sigma_{0}(a)$ denotes the spectrum of $a$ as defined in $C^{*}(a, I)$. Let $C^{*}(a, I) \cong C(X)$ as in Theorem 3.10. For any $\lambda \in \sigma(a), a-\lambda I$ is not invertible so there exists $\phi_{\lambda} \in X$ such that $\phi_{\lambda}(a-\lambda I)=0$, or $\phi_{\lambda}(a)=\lambda$. Conversely, if there is $\phi \in X$ such that $\phi(a)=\lambda$, then $\phi(a-\lambda I)=0$ so $\lambda \in \sigma(a)$. Since any nonzero homomorphism to $\mathbb{C}$ is unital, an element $\phi \in X$ is determined entirely by $\phi(a)$. Since $X$ has the weak* topology, $\phi \mapsto \phi(a)$ is thus a continuous bijection from $X$ to $\sigma(a)$, which is a homeomorphism since $X$ is compact.

It remains to show that $\sigma_{0}(a)=\sigma(a)$. Since an element invertible in the smaller algebra is clearly invertible in the larger algebra, we only need to check that $\sigma(a) \supseteq \sigma_{0}(a)$. Pick $\lambda \in \sigma_{0}(a)$. We need to prove that $a-\lambda I$ is not invertible in $\mathcal{B}(H)$. Assume the contrary and let $b$ be the inverse of $a-\lambda I$. Fix $\epsilon>0$ and let $U \subseteq \sigma_{0}(a)$ be the open ball around $\lambda$ od radius $\epsilon$. Let $g \in C\left(\sigma_{0}(a)\right)$ be a function supported by $U$ such that $\|g\|=1$. Then $g=b(a-\lambda I) g$, hence $\|b(a-\lambda I) g\|=1$. On the other hand, $(a-\lambda I) g=f \in$ $C\left(\sigma_{0}(a)\right)$ so that $f$ vanishes outside of $U$ and $\|f(x)\|<\epsilon$ for $x \in U$, hence $\|(a-\lambda I) g\|<\epsilon$. Thus $\|b\|>1 / \epsilon$ for every $\epsilon>0$, a contradiction.

A spectrum $\sigma_{A}(a)$ of an element $a$ of an arbitrary $\mathrm{C}^{*}$-algebra $A$ can be defined as

$$
\sigma_{A}(a)=\{\lambda \in \mathbb{C} \mid a-\lambda I \text { is not invertible in } A\} .
$$

Lemma 3.14. Suppose $A$ is a unital subalgebra of $B$ and $a \in A$ is normal. Then $\sigma_{A}(a)=\sigma_{B}(a)$, where $\sigma_{A}(a)$ and $\sigma_{B}(a)$ denote the spectra of $a$ as an element of $A$ and $B$, respectively.

Proof. See e.g., [45, Corollary 4.3.16] or [9, Corollary 2 on p. 49].

Note that the isomorphism above is canonical and maps $a$ to the identity function on $\sigma(a)$. It follows that for any polynomial $p$, the isomorphism maps $p(a)$ to the function $z \mapsto p(z)$. More generally, for any continuous function $f: \sigma(a) \rightarrow \mathbb{C}$, we can then define $f(a) \in C^{*}(a, I)$ as the preimage of $f$ under the isomorphism. For example, we can define $|a|$ and if $a$ is self-adjoint then it can be written as a difference of two positive operators as

$$
a=\frac{|a|+a}{2}-\frac{|a|-a}{2} .
$$

If $a \geq 0$, then we can also define $\sqrt{a}$. Here is another application of the "continuous functional calculus" of Corollary 3.13.

Lemma 3.15. Every $a \in \mathcal{B}(H)$ is a linear combination of unitaries.

Proof. By decomposing an arbitrary operator into the positive and negative parts of its real and imaginary parts, it suffices to prove that each positive operator $a$ of norm $\leq 1$ is a linear combination of two unitaries, $u=a+$ $i \sqrt{I-a^{2}}$ and $v=a-i \sqrt{I-a^{2}}$. Clearly $a=\frac{1}{2}(u+v)$. Since $u=v^{*}$ and $u v=v u=I$, the conclusion follows. 
Exercise 3.16. For $a \in \mathcal{B}(H)$ let $a=b u$ be its polar decomposition (see Theorem 2.13).

(1) Show that $b \in C^{*}(a, I)$.

(2) Give an example of $a$ such that $u \notin C^{*}(a, I)$.

(Hint: For (1) use $b=\sqrt{a a^{*}}$. For (2) take $a$ which is compact, but not of finite rank.)

3.4. More examples of $\mathbf{C}^{*}$-algebras. We are now equipped to describe another construction of $\mathrm{C}^{*}$-algebras and more examples.

3.4.1. Direct limits. We now return to giving examples of $\mathrm{C}^{*}$-algebras.

Definition 3.17. If $\Omega$ is a directed set, $A_{i}, i \in \Omega$ are $\mathrm{C}^{*}$-algebras and

$$
\varphi_{i, j}: A_{i} \rightarrow A_{j} \quad \text { for } i<j
$$

is a commuting family of homomorphisms, we define the direct limit (also called the inductive limit) $A=\lim _{i} A_{i}$ by taking the algebraic direct limit and completing it. We define a norm on $A$ by saying that if $a \in A_{i}$,

$$
\|a\|_{A}=\lim _{j}\left\|\varphi_{i, j}(a)\right\|_{A_{j}} .
$$

This limit makes sense because the $\varphi_{i, j}$ are all contractions by Lemma 3.9.

3.4.2. UHF (uniformly hyperfinite) algebras. For each natural number $n$, define a ${ }^{*}$-homomorphism $\Phi_{n}: M_{2^{n}}(\mathbb{C}) \rightarrow M_{2^{n+1}}(\mathbb{C})$ by

$$
\Phi_{n}(a)=\left(\begin{array}{ll}
a & 0 \\
0 & a
\end{array}\right) .
$$

We then define the CAR (Canonical Anticommutation Relations) algebra (aka the Fermion algebra, aka $M_{2}$ UHF algebra) as the direct limit $M_{2}$ = $\lim _{\longrightarrow}\left(M_{2^{n}}(\mathbb{C}), \Phi_{n}\right)$. Alternatively, $M_{2^{\infty}}=\bigotimes_{n \in \mathbb{N}} M_{2}(\mathbb{C})$, since $M_{2^{n+1}}(\mathbb{C})=$ $\vec{M}_{2^{n}}(\mathbb{C}) \otimes M_{2}(\mathbb{C})$ for each $n$ and $\Phi_{n}(a)=a \otimes 1_{M_{2}(\mathbb{C})}$.

Note $\Phi_{n}$ maps diagonal matrices to diagonal matrices, so we can talk about the diagonal elements of $M_{2}$. These turn out to be isomorphic to the algebra $C(K)$, where $K$ is the Cantor set. Thus we can think of $M_{2}$. as a "noncommutative Cantor set."

It is not difficult to see that for $m$ and $n$ in $\mathbb{N}$ there is a unital homomorphism from $M_{m}$ into $M_{n}(\mathbb{C})$ if and only if $m$ divides $n$. If it exists, then this map is unique up to conjugacy. Direct limits of full matrix algebras are called UHF algebras and they were classified by Glimm (the unital case) and Dixmier (the general case) in the 1960s. This was the start of the Elliott classification program of separable unital $\mathrm{C}^{*}$-algebras (see [48], [19]).

The following somewhat laborious exercise is intended to introduce representation theory of the CAR algebra.

Exercise 3.18. Fix $x \in 2^{\mathbb{N}}$ and let $D_{x}=\left\{y \in 2^{\mathbb{N}}:\left(\forall^{\infty} n\right) y(n)=x(n)\right\}$. Enumerate a basis of a complex, infinite-dimensional separable Hilbert space $H$ as $\xi_{y}, y \in D_{x}$. Let $s, t$ range over functions from a finite subset of $\mathbb{N}$ into 
$\{0,1\}$. For such $s$ define a partial isometry of $H$ as follows. If $y(m) \neq s(m)$ for some $m \in \operatorname{dom}(s)$ then let $u_{s}\left(\xi_{y}\right)=0$. Otherwise, if $y\lceil\operatorname{dom}(s)=s$, then let $z \in 2^{\mathbb{N}}$ be such that $z(n)=1-y(n)$ for $n \in \operatorname{dom}(s)$ and $z(n)=y(n)$ for $n \notin \operatorname{dom}(s)$ and set $u_{s}\left(\xi_{y}\right)=\xi_{z}$.

(1) Prove that $u_{s}^{*}=u_{\bar{s}}$, where $\operatorname{dom}(\bar{s})=\operatorname{dom}(s)$ and $\bar{s}(n)=1-s(n)$ for all $n \in \operatorname{dom}(s)$.

(2) Prove that $u_{s} u_{s}^{*}$ is the projection to $\overline{\operatorname{span}}\left\{\xi_{y}: y\lceil\operatorname{dom}(s)=\bar{s}\}\right.$ and $u_{s}^{*} u_{s}$ is the projection to $\overline{\operatorname{span}}\left\{\xi_{y}: y\lceil\operatorname{dom}(s)=s\}\right.$.

(3) Let $A_{x}$ be the $\mathrm{C}^{*}$-algebra generated by $u_{s}$ as defined above. Prove that $A_{x}$ is isomorphic to $M_{2}$.

(4) Show that the intersection of $A_{x}$ with the atomic masa (see $§ 5.1$ ) diagonalized by $\xi_{y}, y \in D_{x}$, consists of all operators of the form $\sum_{y} \alpha_{y} \xi_{y}$ where $y \mapsto \alpha_{y}$ is a continuous function.

(5) Show that for $x$ and $y$ in $2^{\mathbb{N}}$ there is a unitary $v$ of $H$ such that $\operatorname{Ad} v$ sends $A_{x}$ to $A_{y}$ if and only if $\left(\forall^{\infty} n\right) x(n)=y(n)$. (Hint: cf. Example 4.24.)

3.4.3. AF (approximately finite) algebras. Let us start with an exercise. A direct sum of $\mathrm{C}^{*}$-algebras $A$ and $B$ is the algebra $A \oplus B$ whose elements are sums $a+b$ for $a \in A$ and $b \in B$ (assuming $A \cap B=\{0\}$ ), with the convention that $a b=b a=0$ whenever $a \in A$ and $b \in B$. One similarly defines a direct sum of a family of $\mathrm{C}^{*}$-algebras.

Exercise 3.19. Show that a $C^{*}$-algebra $A$ is a finite-dimensional vector space if and only if it is the direct sum of finitely many full matrix algebras.

$\mathrm{A} \mathrm{C}^{*}$-algebra is $\mathrm{AF}$, or approximately finite, if it is a direct limit of finitedimensional $\mathrm{C}^{*}$-algebras. This class of $\mathrm{C}^{*}$-algebras is much more extensive than the class of UHF algebras. Elliott's classification of unital separable $\mathrm{AF}$ algebras by K-theoretic invariant $K_{0}$ (see [48]) marked the beginning of Elliott program for classification of $\mathrm{C}^{*}$-algebras.

Exercise 3.20. Show that an abelian $\mathrm{C}^{*}$-algebra is AF if and only if it is of the form $C_{0}(X)$ for a zero-dimensional, locally compact, Hausdorff, space $X$.

3.4.4. Even more examples. Giving an exhaustive treatment of techniques for building $\mathrm{C}^{*}$-algebras is beyond the scope of this article. Tensor products, group algebras, and crossed products are indispensable tools in theory of $\mathrm{C}^{*}$-algebras. Some of these constructions were blended with set-theoretic methods in [28] to construct novel examples of nonseparable $\mathrm{C}^{*}$-algebras.

\section{Positivity, states and the GNS COnstruction}

The following is a generalization of the spectral theorem to abstract $\mathrm{C}^{*}$ algebras.

Theorem 4.1 (Gelfand-Naimark). Every abelian $C^{*}$-algebra is isomorphic to $C_{0}(X)$ for a unique locally compact Hausdorff space $X$. The algebra is unital if and only if $X$ is compact. 
Proof. By Theorem 3.10, the unitization $B$ of $A$ is isomorphic to $C(\hat{B})$ for a compact Hausdorff space $\hat{B}$, the spectrum of $A$. If $\phi \in \hat{B}$ is the unique map whose kernel is equal to $A$, then $A \cong C_{0}(\hat{B} \backslash\{\phi\})$. The uniqueness of $X=\hat{B} \backslash\{\phi\}$ follows from Theorem 4.17 below.

In fact, the Gelfand-Naimark theorem is functorial: the category of abelian $\mathrm{C}^{*}$-algebras is contravariantly isomorphic to the category of locally compact Hausdorff spaces (cf. Exercise 3.11). The space $X$ is a natural generalization of the spectrum of a single element of a $\mathrm{C}^{*}$-algebra.

Exercise 4.2. (1) If $C^{*}(a)$ is unital and isomorphic to $C(X)$, then $0 \notin$ $\sigma(a)$ and $\sigma(a)$ is homeomorphic to $X$.

(2) If $C^{*}(a)$ is not unital and it is isomorphic to $C_{0}(X)$, then $0 \in \sigma(a)$ and $\sigma(a) \backslash\{0\}$ is homeomorphic to $X$.

Recall that an element $a$ of a $\mathrm{C}^{*}$-algebra $A$ is positive if $a=b^{*} b$ for some $b \in A$. It is not difficult to see that for projections $p$ and $q$ we have $p \leq q$ if and only if $p q=p$ if and only if $q p=p$ (see Lemma 5.5).

Exercise 4.3. Which of the following are true for projections $p$ and $q$ and positive $a$ and $b$ ?

(1) $p q p \leq p$ ?

(2) $a \leq b$ implies $a b=b a$ ?

(3) $p \leq q$ implies $p a p \leq q a q$ ?

(4) $p \leq q$ implies $\operatorname{prp} \leq q r q$ for a projection $r$ ?

(5) $\operatorname{prp} \leq p$ for a projection $r$ ?

(Hint 1: Only one of the above is true. Hint 2: Formula (1) is easy to prove. Hint 3: For (2) note that $a \geq 0$ implies $a \leq a+c$ for every $c \geq 0$. Hint 4: There is a counterexample for (5) on the two-dimensional Hilbert space.)

Definition 4.4. Let $A$ be a $\mathrm{C}^{*}$-algebra. A continuous linear functional $\varphi: A \rightarrow \mathbb{C}$ is positive if $\varphi(a) \geq 0$ for all positive $a \in A$. It is a state if it is positive and of norm 1 . We denote the space of all states on $A$ by $\mathbb{S}(A)$.

Exercise 4.5. Assume $\phi$ is a positive functional on a $\mathrm{C}^{*}$-algebra. Show that $\phi(a)$ is a real whenever $a$ is self-adjoint and show that $\phi\left(b^{*}\right)=\overline{\phi(b)}$ for all $b$. (Hint: Use the continuous function calculus.)

Example 4.6. If $\xi \in H$ is a unit vector, define a functional $\omega_{\xi}$ on $\mathcal{B}(H)$ by

$$
\omega_{\xi}(a)=(a \xi \mid \xi) \text {. }
$$

Then $\omega_{\xi}(a) \geq 0$ for a positive $a$ and $\omega_{\xi}(I)=1$; hence it is a state. We call a state of this form a vector state.

Lemma 4.7. Each positive functional $\phi$ satisfies a Cauchy-Schwartz inequality:

$$
\left|\varphi\left(b^{*} a\right)\right|^{2} \leq \varphi\left(a^{*} a\right) \varphi\left(b^{*} b\right) .
$$


Proof. The proof is similar to the proof of the standard Cauchy-Schwartz inequality. Let $\lambda$ be a complex number. Since $(\lambda a+b)^{*}(\lambda a+b)$ is positive, we have

$$
0 \leq \phi\left((\lambda a+b)^{*}(\lambda a+b)\right)=|\lambda|^{2} \phi\left(a^{*} a\right)+\bar{\lambda} \phi\left(a^{*} b\right)+\lambda \phi\left(b^{*} a\right)+\phi\left(b^{*} b\right) .
$$

We may assume $\left|\phi\left(b^{*} a\right)\right| \neq 0$ since the inequality is trivial otherwise. Let $\lambda=t \phi\left(a^{*} b\right) /\left|\phi\left(b^{*} a\right)\right|$ for a real $t$. Noting that $\phi\left(a^{*} b\right)=\overline{\phi\left(b^{*} a\right)}$ (Exercise 4.5), we obtain

$$
0 \leq t^{2} \frac{\left|\phi\left(a^{*} b\right)\right|^{2}}{\left|\phi\left(b^{*} a\right)\right|^{2}} \phi\left(a^{*} a\right)+t \frac{\overline{\phi\left(a^{*} b\right)}}{\left|\phi\left(b^{*} a\right)\right|} \phi\left(a^{*} b\right)+t \frac{\phi\left(a^{*} b\right)}{\left|\phi\left(b^{*} a\right)\right|} \phi\left(b^{*} a\right)+\phi\left(b^{*} b\right)
$$

or equivalently

$$
0 \leq t^{2} \phi\left(a^{*} a\right)+2 t\left|\phi\left(b^{*} a\right)\right|+\phi\left(b^{*} b\right) .
$$

The discriminant of this equation is nonpositive, and the inequality follows.

Lemma 4.8. If $\varphi$ is a state on $A$ and $0 \leq a \leq I$ is such that $\varphi(a)=1$, then $\varphi(b)=\varphi(a b a)$ for all $b$.

Proof. By the Cauchy-Schwartz inequality for states (Lemma 4.7)

$$
|\varphi((I-a) b)| \leq \sqrt{\varphi(I-a) \varphi\left(b^{*} b\right)}=0 .
$$

Since $b=a b+(I-a) b$, we have $\varphi(b)=\varphi(a b)+\varphi((I-a) b)=\varphi(a b)$. By applying the same argument to $a b$ and multiplying by $I-a$ on the right one proves that $\varphi(a b)=\varphi(a b a)$.

The basic reason we care about states is that they give us representations of abstract $\mathrm{C}^{*}$-algebras as concrete $\mathrm{C}^{*}$-algebras.

Theorem 4.9 (The GNS construction). Let $\varphi$ be a state on $A$. Then there is a Hilbert space $H_{\varphi}$, a representation $\pi_{\varphi}: A \rightarrow \mathcal{B}\left(H_{\varphi}\right)$, and a unit vector $\xi=\xi_{\varphi}$ in $H_{\varphi}$ such that $\varphi=\omega_{\xi} \circ \pi_{\varphi}$.

Skecth of the proof. We define an "inner product" on $A$ by $(a \mid b)=\varphi\left(b^{*} a\right)$. We let $J=\{a:(a \mid a)=0\}$, so that Lemma 4.7 implies $(\cdot \mid \cdot)$ is actually an inner product on the quotient space $A / J$. We then define $H_{\varphi}$ to be the completion of $A / J$ under the induced norm. For any $a \in A, \pi_{\varphi}(a)$ is then the operator that sends $b+J$ to $a b+J$, and $\xi_{\varphi}$ is $I+J$.

4.1. Irreducible representations and pure states. In this section we introduce a particularly important class of states called pure states. The following exercise focuses on a state that is not pure.

Exercise 4.10. Assume $\psi_{1}$ and $\psi_{2}$ are states on $A$ and $0<t<1$ and let

$$
\phi=t \psi_{1}+(1-t) \psi_{2} \text {. }
$$

(1) Show that $\phi$ is a state. 
(2) Show that $H_{\phi} \cong H_{\psi_{1}} \oplus H_{\psi_{2}}$, with $\pi_{\phi}(a)=\pi_{\psi_{1}}(a)+\pi_{\psi_{2}}(a)$, and $\xi_{\phi}=\sqrt{t} \xi_{\psi_{1}}+\sqrt{1-t} \xi_{\psi_{2}}$. In particular, projections to $H_{\psi_{1}}$ and $H_{\psi_{2}}$ commute with $\pi_{\varphi}(a)$ for all $a \in A$.

Hence states form a convex subset of $A^{*}$. We say that a state is pure if it is an extreme point of $\mathbb{S}(A)$. That is, $\varphi$ is pure iff

$$
\varphi=t \psi_{0}+(1-t) \psi_{1}, \quad 0 \leq t \leq 1
$$

for $\psi_{0}, \psi_{1} \in \mathbb{S}(A)$ implies $\varphi=\psi_{0}$ or $\varphi=\psi_{1}$. We denote the set of all pure states on $A$ by $\mathbb{P}(A)$.

Exercise 4.11. Prove the following.

(1) If $\phi$ is a pure state on $M_{n}(\mathbb{C})$ then there is a rank one projection $p$ such that $\phi(a)=\phi($ pap $)$ for all $a$.

(2) Identify $M_{n}(\mathbb{C})$ with $\mathcal{B}\left(\ell_{2}^{n}\right)$. Show that all pure states of $M_{n}(\mathbb{C})$ are vector states.

Recall that the Krein-Milman theorem states that every compact convex subset of a locally convex topological vector space is the closed convex hull of its extreme points. Since the dual space of a $\mathrm{C}^{*}$-algebra is locally convex and since the convex hull of $\mathbb{S}(A) \cup\{0\}$ is compact, we conclude that $\mathbb{S}(A)$ is the weak* closure of the convex hull of $\mathbb{P}(A)$. Since one can show that a $\mathrm{C}^{*}$-algebra has an ample supply of states (see Lemma 4.25 ) the same is true for pure states.

The space $\mathbb{P}(A)$ is weak ${ }^{*}$-compact only for a very restrictive class of $\mathrm{C}^{*}$ algebras, including $\mathcal{K}(H)$ and abelian algebras (see Definition 6.8). For example, for UHF algebras the pure states form a dense subset in the compactum of all states ([33, Theorem 2.8]).

Exercise 4.12. Let $A$ be a separable $\mathrm{C}^{*}$-algebra. Prove that $\mathbb{P}(A)$ is Polish in the weak*-topology.

(Hint: Show that $\mathbb{P}(A)$ is a $G_{\delta}$ subset of $\mathbb{S}(A)$.)

Definition 4.13. A representation $\pi: A \rightarrow \mathcal{B}(H)$ of a $\mathrm{C}^{*}$-algebra is irreducible (sometimes called an irrep) if there is no nontrivial subspace $H_{0} \subset H$ such that $\pi(a) H_{0} \subseteq H_{0}$ for all $a \in A$. Such a subspace is said to be invariant for $\pi[A]$ or reducing for $\pi$.

Theorem 4.14. A state $\varphi$ is pure if and only if $\pi_{\varphi}$ is irreducible. Every irreducible representation is of the form $\pi_{\varphi}$ for some pure state $\varphi$.

Proof. The easy direction is Exercise 4.10. For the other direction see e.g., $[8$, Theorem 1.6.6] or [44, (i) $\Leftrightarrow$ (vi) of Theorem 3.13.2].

Example 4.15. If $A=C(X)$, then by the Riesz representation theorem states are in a bijective correspondence with the Borel probability measures on $X$ (writing $\mu(f)=\int f d \mu$ ).

Exercise 4.16. For a state $\varphi$ of $C(X)$ the following are equivalent:

(1) $\varphi$ is pure, 
(2) for a unique $x_{\varphi} \in X$ we have $\varphi(f)=f\left(x_{\varphi}\right)$

(3) $\varphi: C(X) \rightarrow \mathbb{C}$ is a homomorphism ( $\varphi$ is "multiplicative").

(Hint: Use Example 4.15 and see the proof of Theorem 3.10.)

Theorem 4.17. If $X$ is a compact Hausdorff space then $\mathbb{P}(C(X))$ with respect to the weak ${ }^{*}$-topology is homeomorphic to $X$.

Proof. By (2) in Exercise 4.16, there is a natural map $F: \mathbb{P}(C(X)) \rightarrow X$. By $(3)$, it is not hard to show that $F$ is surjective, and it follows from Urysohn's lemma that $F$ is a homeomorphism.

Proposition 4.18. For any unit vector $\xi \in H$ the vector state $\omega_{\xi} \in \mathbb{S}(\mathcal{B}(H))$ is pure.

Proof. Immediate from Theorem 4.14.

Definition 4.19. We say $\varphi \in \mathbb{S}(\mathcal{B}(H))$ is singular if $\varphi[\mathcal{K}(H)]=\{0\}$.

Exercise 4.20. Prove that a nonsingular state on $\mathcal{B}(H)$ is pure if and only if it is a vector state. (Hint: First show that a nontrivial linear combination of vector states is never pure.)

By factoring through the quotient map $\pi: \mathcal{B}(H) \rightarrow \mathcal{C}(H)$, the space of singular states is isomorphic to the space of states on the Calkin algebra $\mathcal{C}(H)$.

Theorem 4.21. Each state of $\mathcal{B}(H)$ is a weak*-limit of vector states. A pure state is singular if and only if it is not a vector state.

Proof. The first sentence is a special case of [31, Lemma 9] when $\mathfrak{A}=\mathcal{B}(H)$. The second sentence is trivial (modulo Exercise 4.20).

We now take a closer look at the relationship between states and representations of a $\mathrm{C}^{*}$-algebra.

Definition 4.22. Let $A$ be a $\mathrm{C}^{*}$-algebra and $\pi_{i}: A \rightarrow \mathcal{B}\left(H_{i}\right)(i=1,2)$ be representations of $A$. We say $\pi_{1}$ and $\pi_{2}$ are (unitarily) equivalent and write $\pi_{1} \sim \pi_{2}$ if there is a unitary (Hilbert space isomorphism) $u: H_{1} \rightarrow H_{2}$ such that the following commutes:

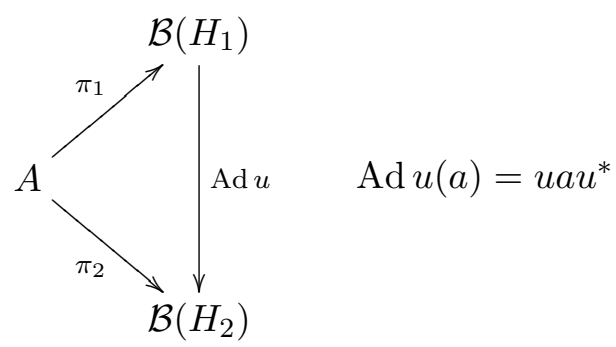


Similarly, if $\varphi_{i} \in \mathbb{P}(A)$, we say $\varphi_{1} \sim \varphi_{2}$ if there is a unitary $u \in \tilde{A}$ such that the following commutes:

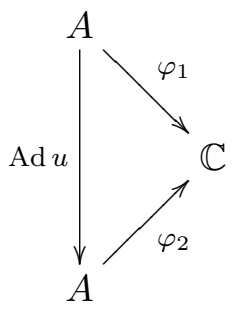

Proposition 4.23. For $\varphi_{i} \in \mathbb{P}(A), \varphi_{1} \sim \varphi_{2}$ if and only if $\pi_{\varphi_{1}} \sim \pi_{\varphi_{2}}$.

Proof. The direct implication is easy and the converse is a consequence of the remarkable Kadison's Transitivity Theorem. For the proof see e.g., [44, the second sentence of Proposition 3.13.4].

4.2. On the existence of states. States on an abelian $\mathrm{C}^{*}$-algebra $C(X)$ correspond to probability Borel measures on $X$ (see Example 4.15).

Example 4.24. On $M_{2}(\mathbb{C})$, the following are pure states:

$$
\begin{array}{rlrl}
\varphi_{0}: & \left(\begin{array}{ll}
a_{11} & a_{12} \\
a_{21} & a_{22}
\end{array}\right) & \mapsto a_{11} \\
\varphi_{1}: \quad\left(\begin{array}{ll}
a_{11} & a_{12} \\
a_{21} & a_{22}
\end{array}\right) \mapsto a_{22}
\end{array}
$$

For any $f \in 2^{\mathbb{N}}, \varphi_{f}=\bigotimes_{n} \varphi_{f(n)}$ is a pure state on $\otimes M_{2}(\mathbb{C})=M_{2}$. Furthermore, one can show that $\varphi_{f}$ and $\varphi_{g}$ are equivalent if and only if $f$ and $g$ differ at only finitely many points, and that $\left\|\varphi_{f}-\varphi_{g}\right\|=2$ for $f \neq g$. See $[44, \S 6.5]$ for a more general setting and proofs.

Lemma 4.25. If $\phi$ is a linear functional of norm 1 on a unital $C^{*}$-algebra then $\phi$ is a state if and only if $\phi(I)=1$.

Proof. First assume $\phi$ is a state. Since $\|\phi\| \leq 1$ and since $\phi$ is positive we have $0 \leq \phi(I) \leq 1$. For positive operators $a \leq b$ we have $\phi(a) \leq \phi(b)$. Since for a positive operator $a$ we have $a \leq\|a\| \cdot I$, we must have $\phi(I)=1$.

Now assume $\phi(I)=\|\phi\|=1$ and fix $a \geq 0$. The algebra $C^{*}(a, I)$ is abelian, and by the Riesz representation theorem the restriction of $\phi$ to this algebra is given by a Borel measure $\mu$ on $\sigma(a)$. The assumption that $\phi(I)=$ $\|\phi\|$ translates as $|\mu|=\mu$, hence $\mu$ is a positive probability measure. Since $a$ corresponds to the identity function on $\sigma(a) \subseteq[0, \infty)$ we have $\phi(a) \geq 0$.

Lemma 4.26. If $A$ is a subalgebra of $B$ then every state of $B$ restricts to a state of $A$. Also, every (pure) state of $A$ can be extended to a (pure) state of $B$.

Proof. The first statement is trivial. Now assume $\phi$ is a state on $A \subseteq B$. We shall extend $\phi$ to a state of $B$ under an additional assumption that $A$ is 
a unital subalgebra of $B$; the general case is then a straightforward exercise (see Lemma 3.2).

By the Hahn-Banach theorem we can extend $\phi$ to a functional $\psi$ on $B$ of norm 1. By Lemma $4.25, \psi$ is a state of $B$.

Note that the (nonempty) set of extensions of $\phi$ to a state of $B$ is weak*compact and convex. If we start with a pure state $\varphi$, then by Krein-Milman the set of extensions of $\varphi$ to $B$ has an extreme point, which can then be shown to be a pure state on $B$.

Lemma 4.27. For every normal $a \in A$ there is a pure state $\phi$ such that $|\phi(a)|=\|a\|$.

Proof. The algebra $C^{*}(a)$ is by Corollary 3.13 isomorphic to $C(\sigma(a))$. Consider its state $\phi_{0}$ defined by $\phi_{0}(f)=f(\lambda)$, where $\lambda \in \sigma(a)$ is such that $\|a\|=|\lambda|$. This is a pure state and satisfies $|\phi(a)|=\|a\|$.

By Lemma 4.25 extend $\phi_{0}$ to a pure state $\phi$ on $A$.

Exercise 4.28. Show that there is a $\mathrm{C}^{*}$-algebra $A$ and $a \in A$ such that $|\phi(a)|<\|a\|$ for every state $\phi$ of $A$.

(Hint: First do Exercise 4.11. Then consider $\left(\begin{array}{ll}0 & 1 \\ 0 & 0\end{array}\right)$ in $M_{2}(\mathbb{C})$.)

Theorem 4.29 (Gelfand-Naimark-Segal). Every $C^{*}$-algebra $A$ is isomorphic to a concrete $C^{*}$-algebra.

Proof. By taking the unitization, we may assume $A$ is unital. Each state $\varphi$ on $A$ gives a representation $\pi_{\varphi}$ on a Hilbert space $H_{\varphi}$, and we take the product of all these representations to get a single representation $\pi=\bigoplus_{\varphi \in \mathbb{S}(A)} \pi_{\varphi}$ on $H=\bigoplus H_{\varphi}$.

We need to check that this representation is faithful. By Lemma 3.9 this is equivalent to $\pi$ being an isometry. By the same Lemma 3.9 we have $\|\pi(a)\| \leq\|a\|$. By Lemma 4.27 for every self-adjoint $a$ we have $|\phi(a)|=\|a\|$.

We claim that $a \neq 0$ implies $\pi(a) \neq 0$. We have that $a=b+i c$ for self-adjoint $b$ and $c$, at least one of which is nonzero. Therefore $\pi(a)=$ $\pi(b)+i \pi(c)$ is nonzero. Thus $A$ is isomorphic to its image $\pi(A) \subseteq \mathcal{B}(H)$, a concrete $\mathrm{C}^{*}$-algebra.

Exercise 4.30. Prove that a separable abstract $\mathrm{C}^{*}$-algebra can be faithfully represented on a separable Hilbert space. (Hint for logicians: LöwenheimSkolem.)

Note that the converse of last exercise is false, since $\mathcal{B}(H)$ itself is nonseparable in norm topology.

Exercise 4.31. (1) Prove that for every $\mathrm{C}^{*}$-algebra $A$ and every $a \in A$ we have

$$
\|a\|^{2}=\sup _{\varphi} \sup _{b} \varphi\left(b^{*} a^{*} a b\right)
$$

where the supremum is taken over all (pure) states $\phi$ and over all $b \in A$ of norm 1 . 
(2) If a $\mathrm{C}^{*}$-algebra $A$ is simple, then for every state $\phi$ and every $a \in A$ we have

$$
\|a\|^{2}=\sup _{b} \varphi\left(b^{*} a^{*} b a\right)
$$

where the supremum is taken over all $b \in A$ of norm 1 .

\section{Projections in the Calkin algebra}

Recall that $\mathcal{K}(H)$ (see Example 3.1.4) is a (norm-closed two-sided) ideal of $\mathcal{B}(H)$, and the quotient $\mathcal{C}(H)=\mathcal{B}(H) / \mathcal{K}(H)$ is the Calkin algebra (see $\S 3.1 .5$ ). We write $\pi: \mathcal{B}(H) \rightarrow \mathcal{C}(H)$ for the quotient map.

Many instances of the question whether an element in a quotient $\mathrm{C}^{*}$ algebra can be lifted to an element with similar properties are well-studied. We shall now consider some of them.

Lemma 5.1. If $\mathbf{a} \in \mathcal{C}(H)$ is self-adjoint, then there is a self-adjoint $a \in$ $\mathcal{B}(H)$ such that $\mathbf{a}=\pi(a)$.

Proof. Fix any $a_{0}$ such that $\pi\left(a_{0}\right)=\mathbf{a}$. Let $a=\left(a_{0}+a_{0}^{*}\right) / 2$. Then $a$ is self-adjoint and $a-a_{0}$ is compact. Therefore $a$ is as required.

Exercise 5.2. Assume $f: A \rightarrow B$ is a *homomorphism between $\mathrm{C}^{*}$-algebras and $p$ is a projection in the range of $f$. Is there necessarily a projection $q \in A$ such that $f(q)=p$ ? (Hint: Consider the natural *-homomorphism from $C([0,1])$ to $C([0,1 / 3] \cup[2 / 3,1])$.)

The following lemma, showing that the answer to question in Exercise 5.2 is sometimes positive, is taken from [58].

Lemma 5.3. If $\mathbf{p} \in \mathcal{C}(H)$ is a projection, then there is a projection $p \in$ $\mathcal{B}(H)$ such that $\mathbf{p}=\pi(p)$.

Proof. Fix a self-adjoint $a$ such that $\mathbf{p}=\pi(a)$. Represent $a$ as a multiplication operator $m_{f}$. Since $\pi\left(m_{f}\right)$ is a projection, $m_{f^{2}-f} \in \mathcal{K}(H)$ Let

$$
h(x)= \begin{cases}1, & f(x) \geq 1 / 2 \\ 0, & f(x)<1 / 2\end{cases}
$$

Then $m_{h}$ is a projection. Also, if $\left(x_{\alpha}\right)$ is such that $f\left(x_{\alpha}\right)^{2}-f\left(x_{\alpha}\right) \rightarrow 0$, then $h\left(x_{\alpha}\right)-f\left(x_{\alpha}\right) \rightarrow 0$. One can show that this implies that since $m_{f^{2}-f}$ is compact, so is $m_{h-f}$. Hence $\pi\left(m_{h}\right)=\pi\left(m_{f}\right)=\mathbf{p}$.

Thus self-adjoints and projections in $\mathcal{C}(H)$ are just self-adjoints and projections in $\mathcal{B}(H)$ modded out by compacts. However, the same is not true for unitaries.

Example 5.4. Let $S \in \mathcal{B}(H)$ be the unilateral shift (Example 2.12). Then $S^{*} S=I$ and $S S^{*}=I-\operatorname{proj}_{\overline{\operatorname{span}}\left(\left\{e_{0}\right\}\right)}=I-p$. Since $p$ has finite-dimensional range, it is compact, so $\pi(S)^{*} \pi(S)=I=\pi(S) \pi\left(S^{*}\right)$. That is, $\pi(S)$ is unitary. 
If $\pi(a)$ is invertible, one can define the Fredholm index of $a$ by

$$
\operatorname{index}(a)=\operatorname{dim} \operatorname{ker} a-\operatorname{dim} \operatorname{ker} a^{*} .
$$

The Fredholm index is (whenever defined) invariant under compact perturbations of $a$ ([45, Theorem 3.3.17]). Since index $(u)=0$ for any unitary $u$ and $\operatorname{index}(S)=-1$, there is no unitary $u \in \mathcal{B}(H)$ such that $\pi(u)=\pi(S)$.

For a $\mathrm{C}^{*}$-algebra $A$ we write $\mathcal{P}(A)$ for the set of projections in $A$. We partially order $\mathcal{P}(A)$ by saying $p \leq q$ if $p q=p$. This agrees with the restriction of the ordering on positive operators. If they exist, we denote joins and meets under this ordering by $p \vee q$ and $p \wedge q$. Note that every $p \in \mathcal{P}(A)$ has a canonical (orthogonal) complement $q=I-p$ such that $p \vee q=I$ and $p \wedge q=0$.

Lemma 5.5. Let $p, q \in A$ be projections. Then $p q=p$ if and only if $q p=p$.

Proof. Since $p=p^{*}$ and $q=q^{*}$, if $p q=p$ then $p q=(p q)^{*}=q^{*} p^{*}=q p$. The converse is similar.

Lemma 5.6. Let $p, q \in A$ be projections. Then $p q=q p$ if and only if $p q$ is a projection, in which case $p q=p \wedge q$ and $p+q-p q=p \vee q$.

Proof. If $p q=q p,(p q)^{*}=q^{*} p^{*}=q p=p q$ and $(p q)^{2}=p(q p) q=p^{2} q^{2}=p q$. Conversely, if $p q$ is a projection then $q p=(p q)^{*}=p q$. Clearly then $p q \leq p$ and $p q \leq q$, and if $r \leq p$ and $r \leq q$ then $r p q=(r p) q=r q=r$ so $r \leq p q$. Hence $p q=p \wedge q$. We similarly have $(1-p)(1-q)=(1-p) \wedge(1-q)$; since $r \mapsto 1-r$ is an order-reversing involution it follows that $p+q-p q=$ $1-(1-p)(1-q)=p \vee q$.

For $A=\mathcal{B}(H)$, note that $p \leq q$ if and only if $\operatorname{range}(p) \subseteq \operatorname{range}(q)$. Also, joins and meets always exist in $\mathcal{B}(H)$ and are given by

$$
\begin{gathered}
p \wedge q=\text { the projection onto range }(p) \cap \operatorname{range}(q), \\
p \vee q=\text { the projection onto } \overline{\operatorname{span}}(\operatorname{range}(p) \cup \operatorname{range}(q)) .
\end{gathered}
$$

That is, $\mathcal{P}(\mathcal{B}(H))$ is a lattice (in fact, it is a complete lattice, as the definitions of joins and meets above generalize naturally to infinite joins and meets).

Note that if $X$ is a connected compact Hausdorff space then $C(X)$ has no projections other than 0 and $I$.

Proposition 5.7. $\mathcal{B}(H)=C^{*}(\mathcal{P}(\mathcal{B}(H)))$. That is, $\mathcal{B}(H)$ is generated by its projections.

Proof. Since every $a \in \mathcal{B}(H)$ is a linear combination of self-adjoints $a+a^{*}$ and $i\left(a-a^{*}\right)$, it suffices to show that if $b$ is self-adjoint and $\epsilon>0$ then there is a linear combination of projections $c=\sum_{j} \alpha_{j} p_{j}$ such that $\|b-c\|<$ $\epsilon$. For this we may use spectral theorem and approximate $m_{f}$ by a step function. 
Corollary 5.8. $\mathcal{C}(H)=C^{*}(\mathcal{P}(\mathcal{C}(H)))$. That is, $\mathcal{C}(H)$ is generated by its projections.

Proof. Since a *-homomorphism sends projections to to projections, this is a consequence of Proposition 5.7

Proposition 5.9. Let $A$ be an abelian unital $C^{*}$-algebra. Then $\mathcal{P}(A)$ is a Boolean algebra.

Proof. By Lemma 5.6, commuting projections always have joins and meets, and $p \mapsto I-p$ gives complements. It is then easy to check that this is actually a Boolean algebra using the formulas for joins and meets given by Lemma 5.6.

5.0.1. Stone duality. Let us recall the Stone duality for Boolean algebras. For a Boolean algebra $\mathbb{B}$ its Stone space Stone $(\mathbb{B})$ is the compact Hausdorff space of all ultrafilters of $\mathbb{B}$ with the topology generated by its basic open sets $U_{a}=\{\mathcal{U} \in \operatorname{Stone}(\mathbb{B}): a \in \mathcal{U}\}$, for $a \in \mathbb{B} \backslash\left\{0_{\mathbb{B}}\right\}$. It is well-known that the algebra of clopen subsets of $\operatorname{Stone}(\mathbb{B})$ is isomorphic to $\mathbb{B}$. Also, to every Boolean algebra homomorphism $\Phi: \mathbb{B}_{1} \rightarrow \mathbb{B}_{2}$ one associates a continuous map $f_{\Phi}:$ Stone $\left(\mathbb{B}_{2}\right) \rightarrow \operatorname{Stone}\left(\mathbb{B}_{1}\right)$, so that $f_{\Phi}(\mathcal{U})=\Phi^{-1}(\mathcal{U})$ for all $\mathcal{U} \in \operatorname{Stone}\left(\mathbb{B}_{2}\right)$. Conversely, if $f: \operatorname{Stone}\left(\mathbb{B}_{2}\right) \rightarrow \operatorname{Stone}\left(\mathbb{B}_{1}\right)$ is a continuous map, then $\Phi_{f}(a)=b$ for $b$ such that $f^{-1}\left(U_{a}\right)=U_{b}$ is a Boolean algebra homomorphism. It is straightforward to show that (i) the operations $f \mapsto \Phi_{f}$ and $\Phi \mapsto f_{\Phi}$ are inverses of one another, (ii) $f$ is a surjection if and only if $\Phi_{f}$ is an injection, and (iii) $f$ is an injection if and only if $\Phi_{f}$ is a surjection. Altogether this shows that the category of compact zero-dimensional Hausdorff spaces is contravariantly equivalent to the category of Boolean algebras.

By combining Stone duality with Gelfand-Naimark theorem (see the remark after Theorem 4.1) one obtains isomorphism between the categories of Boolean algebras and abelian $\mathrm{C}^{*}$-algebras generated by their projections.

Note that if $A$ is nonabelian, then even if $\mathcal{P}(A)$ is a lattice it may be nondistributive and hence not a Boolean algebra. See also Proposition 5.26 below.

Exercise 5.10. Prove that the following are equivalent for a $\mathrm{C}^{*}$-algebra $A$.

(1) The set of all invertible self-adjoint elements of $A$ is dense in the set of all self-adjoint elements of $A$.

(2) The set of all linear combinations of projections is dense in $A$.

$\mathrm{C}^{*}$-algebras satisfying conditions of Exercise 5.10 are said to have real rank zero.

Exercise 5.11. Prove that $C(X)$ has real rank zero if and only if $X$ is zero-dimensional. 
5.1. Maximal abelian subalgebras. In this section we will be interested in abelian (unital) subalgebras of $\mathcal{B}(H)$ and $\mathcal{C}(H)$. In particular, we will look at maximal abelian subalgebras, or "masas." The acronym masa stands for 'Maximal Abelian SubAlgebra' or 'MAximal Self-Adjoint subalgebra.' Pedersen ([45]) uses MAÇA, for 'MAximal Commutative subAlgebra.' ${ }^{2}$ Note that if $H=L^{2}(X, \mu)$, then $L^{\infty}(X, \mu)$ is an abelian subalgebra of $\mathcal{B}(H)$ (as multiplication operators).

Theorem 5.12. $L^{\infty}(X, \mu) \subset \mathcal{B}\left(L^{2}(X, \mu)\right)$ is a masa.

Proof. See [9, Theorem 4.1.2] or [45, Theorem 4.7.7].

Conversely, every masa in $\mathcal{B}(H)$ is of this form. To prove this, we need a stronger form of the spectral theorem, which applies to abelian subalgebras rather than just single normal operators.

Theorem 5.13 (General Spectral Theorem). If $A$ is an abelian subalgebra of $\mathcal{B}(H)$ then there is a probability measure space $(X, \mu)$, a subalgebra $B$ of $L^{\infty}(X, \mu)$, and a Hilbert space isomorphism $\Phi: L^{2}(X, \mu) \rightarrow H$ such that $\operatorname{Ad} \Phi[B]=A$.

Proof. See [9, Theorem 4.7.13].

Corollary 5.14. For any masa $A \subset \mathcal{B}(H)$, there is a probability measure space $(X, \mu)$ and a Hilbert space isomorphism $\Phi: L^{2}(X, \mu) \rightarrow H$ such that

$$
\operatorname{Ad} \Phi\left[L^{\infty}(X, \mu)\right]=A .
$$

Proof. By maximality, $B$ must be all of $L^{\infty}(X)$ in the spectral theorem.

Corollary 5.14 can be used to classify masas in $\mathcal{B}(H)$. The two most important examples of masas are given in the following two examples.

Example 5.15 (Atomic masa in $\mathcal{B}(H)$ ). Fix an orthonormal basis $\left(e_{n}\right)$ for $H$, which gives an identification $H \cong \ell^{2}(\mathbb{N})=\ell^{2}$. The corresponding masa is then $\ell^{\infty}$, or all operators that are diagonalized by the basis $\left(e_{n}\right)$. We call this an atomic masa because the corresponding measure space is atomic. The projections in $\ell^{\infty}$ are exactly the projections onto subspaces spanned by a subset of $\left\{e_{n}\right\}$. That is, $\mathcal{P}\left(\ell^{\infty}\right) \cong \mathcal{P}(\mathbb{N})$. In particular, if we fix a basis, then the Boolean algebra $\mathcal{P}(\mathbb{N})$ is naturally a sublattice of $\mathcal{P}(\mathcal{B}(H))$. Given $X \subseteq \mathbb{N}$, we write $P_{X}^{(\vec{e})}$ for the projection onto $\overline{\operatorname{span}}\left\{e_{n}: n \in X\right\}$.

Exercise 5.16. Prove that the atomic masa is isomorphic to $C(\beta \mathbb{N})$, where $\mathbb{N}$ is the Cech-Stone compactification of $\mathbb{N}$ taken with the discrete topology. (Hint: Cf. Exercise 3.12.)

Example 5.17 (Atomless masa in $\mathcal{B}(H)$ ). Let $(X, \mu)$ be any atomless probability measure space. Then if we identify $H$ with $L^{2}(X), L^{\infty}(X) \subseteq \mathcal{B}(H)$ is the atomless masa. The projections in $L^{\infty}(X)$ are exactly the characteristic

\footnotetext{
${ }^{2}$ This acronym acquires a whole new meaning in light of the related ASHCEFLC ([44, $6.2 .13])$
} 
functions of measurable sets, so $\mathcal{P}\left(L^{\infty}(X)\right)$ is the measure algebra of $(X, \mu)$ (modulo the null sets).

Proposition 5.18. Let $\mathcal{A} \subseteq \mathcal{B}(H)$ be an atomless masa. Then $\mathcal{P}(\mathcal{A})$ is isomorphic to the Lebesgue measure algebra of measurable subsets of $[0,1]$ modulo null sets.

Proof. Omitted, but see the remark following Proposition 5.9.

We now relate masas in $\mathcal{B}(H)$ to masas in $\mathcal{C}(H)$.

Theorem 5.19 (Johnson-Parrott, $1972[36]$ ). If $\mathcal{A}$ is a masa in $\mathcal{B}(H)$ then $\pi[\mathcal{A}]$ is a masa in $\mathcal{C}(H)$.

Proof. Assume $b \in \mathcal{B}(H)$ is such that $\pi(b)$ belongs to the commutant of $\pi[\mathcal{A}]$. We need to find $a \in \mathcal{A}$ such that $a-b$ is compact. Consider the map $\delta_{b}: \mathcal{A} \rightarrow \mathcal{B}(H)$ defined by

$$
\delta_{b}(x)=b x-x b .
$$

Then $\delta_{b}(x)$ is compact for every $a \in \mathcal{A}$. A straightforward computation shows that $\delta_{b}(x y)=\delta_{b}(x) y+x \delta_{b}(y)$. Such a map is called a derivation. By [36, Theorem 2.1], every derivation from the atomic masa into $\mathcal{B}(H)$ is trivial, i.e., of the form $\delta_{a}$ for some $a$ in the atomic masa. Then $a \in \mathcal{A}$ such that $\delta_{a}$ and $\delta_{b}$ agree on $\mathcal{A}$ is as required, by [36, Lemma 1.4].

Theorem 5.20 (Akemann-Weaver [2]). There exists a masa $A$ in $\mathcal{C}(H)$ that is not of the form $\pi[\mathcal{A}]$ for any masa $\mathcal{A} \subset \mathcal{B}(H)$.

Proof. By Corollary 5.14, each masa in $\mathcal{B}(H)$ is induced by an isomorphism from $H$ to $L^{2}(X)$ for a probability measure space $X$. But the measure algebra of a probability measure space is countably generated, so there are only $2^{\aleph_{0}}$ isomorphism classes of probability measure spaces. Since $H$ is separable, it follows that there are at most $2^{\aleph_{0}}$ masas in $\mathcal{B}(H)$.

Now fix an almost disjoint (modulo finite) family $\mathbb{A}$ of infinite subsets of $\mathbb{N}$ of size $2^{\aleph_{0}}$. Recall that $P_{X}^{(\vec{e})}$ is the projection to the closed subspace spanned by $\left\{e_{n}: n \in X\right\}$. Then the projections $p_{X}=\pi\left(P_{X}^{(\vec{e})}\right)$, for $X \in \mathbb{A}$, form a family of orthogonal projections in $\mathcal{C}(H)$. Choose non-commuting projections $q_{X, 0}$ and $q_{X, 1}$ in $\mathcal{C}(H)$ below $p_{X}$. To each $f: \mathbb{A} \rightarrow\{0,1\}$ associate a family of orthogonal projections $\left\{q_{X, f(X)}\right\}$. Extending each of these families to a masa, we obtain $2^{2^{\aleph_{0}}}$ distinct masas in $\mathcal{C}(H)$. Therefore some masa in $\mathcal{C}(H)$ is not of the form $\pi[\mathcal{A}]$ for any masa in $\mathcal{B}(H)$.

Anderson ([5]) asked whether there is a masa in the Calkin algebra that is generated by projections but not of the form $\pi[\mathcal{A}]$ for some masa $\mathcal{A}$ in $\mathcal{B}(H)$. Note that this question is not answered by Theorem 5.20 since masa constructed there are not necessarily generated by their projections. By [52] very mild set-theoretic assumptions imply the existence of such masa. It is not known whether this can be proved in ZFC. 
Lemma 5.21. Let $\mathcal{A} \subset \mathcal{B}(H)$ be a masa. Then $J=\mathcal{P}(\mathcal{A}) \cap \mathcal{K}(H)$ is a Boolean ideal in $\mathcal{P}(\mathcal{A})$ and $\mathcal{P}(\pi[\mathcal{A}])=\mathcal{P}(\mathcal{A}) / J$.

Proof. It is easy to check that $J$ is an ideal since $\mathcal{K}(H) \subseteq \mathcal{B}(H)$ is an ideal. Let $a \in \mathcal{A}$ be such that $\pi(a)$ is a projection. Writing $\mathcal{A}=L^{\infty}(X)$, then in the proof of Lemma 5.3, we could have chosen to represent $a$ as a multiplication operator on $L^{2}(X)$, in which case the projection $p$ that we obtain such that $\pi(p)=\pi(a)$ is also a multiplication operator on $L^{2}(X)$. That is there is a projection $p \in \mathcal{A}$ such that $\pi(p)=\pi(a)$. Thus $\pi: \mathcal{P}(\mathcal{A}) \rightarrow \mathcal{P}(\pi[\mathcal{A}])$ is surjective. Furthermore, it is clearly a Boolean homomorphism and its kernel is $J$, so $\mathcal{P}(\pi[\mathcal{A}])=\mathcal{P}(\mathcal{A}) / J$.

Exercise 5.22. Let $\mathcal{A}$ be the CAR algebra ( $\S 3.4 .2)$ and let $\mathcal{D}$ be its subalgebra generated by the diagonal matrices. Show that $\mathcal{D}$ is a masa in $\mathcal{A}$.

5.2. Projections in the Calkin algebra. In the present section we study the poset of projections in the Calkin algebra. This structure is closely related to the Boolean algebra $\mathcal{P}(\mathbb{N}) /$ Fin, although in many ways it is closer to quotients over analytic P-ideals such as the asymptotic density zero ideal, $\mathcal{Z}_{0}$.

Lemma 5.23. A projection $p \in \mathcal{B}(H)$ is compact if and only if its range is finite-dimensional.

Proof. If we let $B \subseteq H$ be the unit ball, $p$ is compact if and only if $p[B]$ is precompact. But $p[B]$ is just the unit ball in the range of $p$, which is (pre)compact if and only if the range is finite-dimensional.

Let us now take a closer look at the images of the two distinguished masas in $\mathcal{B}(H)$.

If $\mathcal{A}=\ell^{\infty}$ is an atomic masa in $\mathcal{B}(H)$, then we obtain an "atomic" masa $\pi[\mathcal{A}]$ in $\mathcal{C}(H)$. By Lemmas 5.21 and $5.23, \mathcal{P}(\pi[\mathcal{A}]) \cong \mathcal{P}(\mathbb{N}) /$ Fin, where Fin is the ideal of finite sets. In particular, if we fix a basis then $\mathcal{P}(\mathbb{N}) /$ Fin naturally embeds in $\mathcal{P}(\mathcal{C}(H))$. For this reason, we can think of $\mathcal{P}(\mathcal{C}(H))$ as a "noncommutative" version of $\mathcal{P}(\mathbb{N}) /$ Fin. Moreover, one can show that $\mathcal{A} \cap \mathcal{K}(H)=c_{0}$, the set of sequences converging to 0 , so that $\pi[\mathcal{A}]=\ell^{\infty} / c_{0}=$ $C(\beta \mathbb{N} \backslash \mathbb{N})$.

If $\mathcal{A}$ is an atomless masa in $\mathcal{B}(H)$, then all of its projections are infinitedimensional. Thus $\mathcal{P}(\pi[\mathcal{A}])=\mathcal{P}(\mathcal{A})$. Thus the Lebesgue measure algebra also embeds in $\mathcal{P}(\mathcal{C}(H))$.

Lemma 5.24. For projections $p$ and $q$ in $\mathcal{B}(H)$, the following are equivalent:

(1) $\pi(p) \leq \pi(q)$,

(2) $p(I-q)$ is compact,

(3) For any $\epsilon>0$, there is a finite-dimensional projection $p_{0} \leq I-p$ such that $\left\|q\left(I-p-p_{0}\right)\right\|<\epsilon$.

Proof. The equivalence of (1) and (2) is trivial. For the remaining part see [58, Proposition 3.3]. 
We write $p \leq_{\mathcal{K}} q$ if the conditions of Lemma 5.24 are satisfied. The poset $(\mathcal{P}(\mathcal{C}(H)), \leq)$ is then isomorphic to the quotient $\left(\mathcal{P}(\mathcal{B}(H)), \leq_{\mathcal{K}}\right) / \sim$, where $p \sim q$ if $p \leq_{\mathcal{K}} q$ and $q \leq_{\mathcal{K}} p$. In the strong operator topology, $\mathcal{P}(\mathcal{B}(H))$ is Polish, and (3) in Lemma 5.24 then implies that $\leq_{\mathcal{K}} \subset \mathcal{P}(\mathcal{B}(H)) \times \mathcal{P}(\mathcal{B}(H))$ is Borel.

Lemma 5.25. There are projections $p$ and $q$ in $\mathcal{B}(H)$ such that $\pi(p)=$ $\pi(q) \neq 0$ but $p \wedge q=0$.

Proof. Fix an orthonormal basis $\left(e_{n}\right)$ for $H$ and let $\alpha_{n}=1-\frac{1}{n}$ and $\beta_{n}=$ $\sqrt{1-\alpha_{n}^{2}}$. Vectors $\xi_{n}=\alpha_{n} e_{2 n}+\beta_{n} e_{2 n+1}$ for $n \in \mathbb{N}$ are orthonormal and they satisfy $\lim _{n}\left(\xi_{n} \mid e_{2 n}\right)=1$. Projections $p=\operatorname{proj}_{\overline{\operatorname{span}}\left\{e_{2 n}: n \in \mathbb{N}\right\}}$ and $q=$ $\operatorname{proj}_{\overline{\operatorname{span}}\left\{\xi_{n}: n \in \mathbb{N}\right\}}$ are as required.

Recall that $\mathcal{P}(\mathcal{B}(H))$ is a complete lattice, which is analogous to the fact that $\mathcal{P}(\mathbb{N})$ is a complete Boolean algebra. Since $\mathcal{P}(\mathbb{N}) /$ Fin is not a complete Boolean algebra, we would not expect $\mathcal{P}(\mathcal{C}(H))$ to be a complete lattice. More surprisingly, however, the "noncommutativity" of $\mathcal{P}(\mathcal{C}(H))$ makes it not even be a lattice at all.

Proposition 5.26 (Weaver). $\mathcal{P}(\mathcal{C}(H))$ is not a lattice.

Proof. Enumerate an orthogonal basis of $H$ as $\left\{\xi_{m n}, \eta_{m n}: m \in \mathbb{N}, n \in \mathbb{N}\right\}$. Define

$$
\zeta_{m n}=\frac{1}{n} \xi_{m n}+\frac{\sqrt{n-1}}{n} \eta_{m n}
$$

and

$$
\begin{aligned}
K & =\overline{\operatorname{span}}\left\{\eta_{m n}: m, n \in \mathbb{N}\right\}, & & p=\operatorname{proj}_{K} \\
L & =\overline{\operatorname{span}}\left\{\zeta_{m n}: m, n \in \mathbb{N}\right\}, & & q=\operatorname{proj}_{L} .
\end{aligned}
$$

For $f: \mathbb{N} \rightarrow \mathbb{N}$, define

$$
M(f)=\overline{\operatorname{span}}\left\{\eta_{m n}: m \leq f(n)\right\} \text { and } r(f)=\operatorname{proj}_{M(f)} .
$$

It is easy to show, using Lemma 5.24 , that $r(f) \leq p$ and $r(f) \leq_{\mathcal{K}} q$ for all $f$, and that if $f<g$ then $r(f)<\mathcal{K} r(g)$ strictly.

Now assume $r$ is a projection such that $r \leq_{\mathcal{K}} p$ and $r \leq_{\mathcal{K}} q$. Again using Lemma 5.24 one sees that $r \leq_{\mathcal{K}} r(f)$ for some $f$. In particular, it follows that $p$ and $q$ cannot have a meet under $\leq \mathcal{K}$.

5.3. Cardinal invariants. Since cardinal invariants can often be defined in terms of properties of subsets of $\mathcal{P}(\mathbb{N}) /$ Fin (see [13]), we can look for "noncommutative" (or "quantum") versions of cardinal invariants by looking at analogous properties of $\mathcal{P}(\mathcal{C}(H))$.

Recall that $\mathfrak{a}$ denotes the minimal possible cardinality of a maximal infinite antichain in $\mathcal{P}(\mathbb{N}) /$ Fin, or equivalently the minimal possible cardinality of an (infinite) maximal almost disjoint family in $\mathcal{P}(\mathbb{N})$. 
Definition 5.27 (Wofsey, [59]). A family $\mathbb{A} \subseteq \mathcal{P}(\mathcal{B}(H)$ ) is almost orthogonal (ao) if $p q$ is compact for $p \neq q$ in $\mathbb{A}$ but no $p \in \mathbb{A}$ is compact. We define $\mathfrak{a}^{*}$ to be the minimal possible cardinality of an infinite maximal ao family ("mao family").

Note that we require every $p \in \mathbb{A}$ to be noncompact since while Fin $\subset$ $\mathcal{P}(\mathbb{N})$ is only countable, there are $2^{\aleph_{0}}$ compact projections in $\mathcal{P}(\mathcal{B}(H))$.

Theorem 5.28 (Wofsey, [59]). (1) It is relatively consistent with ZFC that $\aleph_{1}=\mathfrak{a}=\mathfrak{a}^{*}<2^{\aleph_{0}}$,

(2) MA implies $\mathfrak{a}^{*}=2^{\aleph_{0}}$.

Proof. Omitted.

Question 5.29. Is $\mathfrak{a}=\mathfrak{a}^{*}$ ? Is $\mathfrak{a} \geq \mathfrak{a}^{*}$ ? Is $\mathfrak{a}^{*} \geq \mathfrak{a}$ ?

It may seem easy to prove that $\mathfrak{a} \geq \mathfrak{a}^{*}$, since $\mathcal{P}(\mathbb{N}) /$ Fin embeds in $\mathcal{P}(\mathcal{C}(H))$ so any maximal almost disjoint family would give a mao family. However, it turns out that a maximal almost disjoint family can fail to be maximal as an almost orthogonal family. We now proceed to give an example of such a family.

An ideal $J$ on $\mathcal{P}(\mathbb{N})$ is a $p$-ideal if for every sequence $X_{n}, n \in \mathbb{N}$ of elements of $J$ there is $X \in J$ such that $X_{n} \backslash X$ is finite for all $n$.

Lemma 5.30 (Steprāns, 2007). Fix $a \in \mathcal{B}(H)$ and a basis $\left(e_{n}\right)$ for $H$. Then

$$
J_{a}=\left\{X \subseteq \mathbb{N}: P_{X}^{(\vec{e})} a \text { is compact }\right\}
$$

is a Borel P-ideal.

Proof. Let $\varphi_{a}(X)=\left\|P_{X} a\right\|$. This is a lower semicontinuous submeasure on $\mathbb{N}$, and $P_{X} a$ is compact if and only if $\lim _{n} \varphi_{a}(X \backslash n)=0$ (see equivalent conditions (1)-(3) in Example 3.1.4). Thus $J_{a}$ is $F_{\sigma \delta}$. Proving that it is a p-ideal is an easy exercise.

Proposition 5.31 (Wofsey, [59]). There is a maximal almost disjoint family $\mathbb{A} \subset \mathcal{P}(\mathbb{N})$ whose image in $\mathcal{P}(\mathcal{B}(H))$ is not a mao family.

Proof. Let

$$
\xi_{n}=2^{-n / 2} \sum_{j=2^{n}}^{2^{n+1}-1} e_{j} .
$$

Then $\xi_{n}$, for $n \in \mathbb{N}$, are orthonormal and $q=\operatorname{proj}_{\overline{\operatorname{span}}\left\{\xi_{n}\right\}}$. Since $\lim _{n}\left\|q e_{n}\right\|=$ 0 the ideal $J_{q}$ is dense: every infinite subset of $\mathbb{N}$ has an infinite subset in $J_{q}$ (choose a sparse enough subset $X$ such that $\sum_{n \in X}\left\|q e_{n}\right\|<\infty$ ). By density, we can find a maximal almost disjoint family $\mathbb{A}$ that is contained in $J_{q}$. Then $q$ is almost orthogonal to $P_{X}$ for all $X \in \mathbb{A}$, so $\left\{P_{X}: X \in \mathbb{A}\right\}$ is not a mao family.

In some sense, this is the only way to construct such a counterexample. More precisely, we have the following: 
Theorem 5.32. Let $\mathfrak{a}^{\prime}$ denote the minimal possible cardinality of a maximal almost disjoint family that is not contained in any proper Borel P-ideal. Then $\mathfrak{a}^{\prime} \geq \mathfrak{a}$ and $\mathfrak{a}^{\prime} \geq \mathfrak{a}^{*}$.

Proof. The inequality $\mathfrak{a}^{\prime} \geq \mathfrak{a}$ is trivial, and the inequality $\mathfrak{a}^{\prime} \geq \mathfrak{a}^{*}$ follows by Lemma 5.30.

One can also similarly define other quantum cardinal invariants: $\mathfrak{p}^{*}, \mathfrak{t}^{*}, \mathfrak{b}^{*}$, etc (see e.g., [13]). For example, recall that $\mathfrak{b}$ is the minimal cardinal $\kappa$ such that there exists a $(\kappa, \omega)$-gap in $\mathcal{P}(\mathbb{N}) /$ Fin and let $\mathfrak{b}^{*}$ be the minimal cardinal $\kappa$ such that there exists a $(\kappa, \omega)$-gap in $\mathcal{P}(\mathcal{C}(H))$. Considerations similar to those needed in the proof of Proposition 5.26 lead to following.

Theorem 5.33 (Zamora-Avilés, [60]). $\mathfrak{b}=\mathfrak{b}^{*}$.

Proof. Omitted.

Almost all other questions about the relationship between these and ordinary cardinal invariants are open. One should also note that equivalent definitions of standard cardinal invariants may lead to distinct quantum cardinal invariants.

5.4. A twist of projections. A question that may be related to cardinal invariants is when collections of commuting projections of $\mathcal{C}(H)$ can be simultaneously lifted to $\mathcal{B}(H)$ such that the lifts still commute. Let $\mathfrak{l}$ (this symbol is \mathfrak 1 ) be the minimal cardinality of such a collection that does not lift. From the proof of Theorem 5.20 it follows that such collections exist. Note that if instead of projections in the definition of $\mathfrak{l}$ we consider arbitrary commuting operators, then the value of a cardinal invariant defined in this way drops to 2. To see this, consider the unilateral shift and its adjoint (see Example 5.4).

Lemma 5.34. The cardinal $\mathfrak{l}$ is uncountable. Given any sequence $p_{i}$ of projections in $\mathcal{B}(H)$ such that $\pi\left(p_{i}\right)$ and $\pi\left(p_{j}\right)$ commute for all $i, j$, there is an atomic masa $\mathcal{A}$ in $\mathcal{B}(H)$ such that $\pi[\mathcal{A}]$ contains all $\pi\left(p_{i}\right)$.

Proof. Let $\zeta(i), i \in \mathbb{N}$, be a norm-dense subset of the unit ball of $H$. We will recursively choose projections $q_{i}$ in $\mathcal{B}(H)$, orthonormal basis $e_{i}$, and $k(j) \in \mathbb{N}$ so that for all $i \leq k(j)$ we have $\pi\left(q_{i}\right)=\pi\left(p_{i}\right), q_{i}\left(e_{j}\right) \in\left\{e_{j}, 0\right\}$ and $\zeta(j)$ is in the span of $\left\{e_{i}: i<k(j)\right\}$. Assume $q_{j}, j<n$, and $e_{i}, i<k(n)$, have been chosen to satisfy these requirements. Let $r$ be the projection to the orthogonal complement of $\left\{e_{i} \mid i<k(n)\right\}$ and for each $\alpha \in\{1, \perp\}^{n}$ let $r_{\alpha}=r \prod_{i<n} q_{i}^{\alpha(i)}$. For each $\alpha \in\{1, \perp\}^{n}$ we have that $\pi\left(p_{n}\right)$ and $\pi\left(r_{\alpha}\right)$ commute, hence by Lemma 5.3 there is a projection $p_{\alpha}$ in $\mathcal{B}\left(r_{\alpha}[H]\right)$ such that $\pi\left(p_{\alpha}\right)=\pi\left(p_{n}\right) \pi\left(r_{\alpha}\right)$, and $\pi\left(p_{n}\right)=\sum_{\alpha} \pi\left(p_{n}\right) \pi\left(r_{\alpha}\right)$. Note that we have

$$
q_{n}=\sum_{\alpha \in\{1, \perp\}^{n}} p_{\alpha} \text {. }
$$

Now pick $k(n+1)$ large enough and unit vectors $e_{i}, k(n) \leq i<k(n+1)$, each belonging in some $r_{\alpha} q_{n}[H]$, such that $e_{i}, i<k(n+1)$ span $\zeta(n)$. 
This assures $\left(e_{i}\right)$ is a basis of $H$. Let $X(i)=\{n: n \geq k(i)$ and the unique $\alpha \in\{1, \perp\}^{n}$ such that $e_{n} \in r_{\alpha}(n)$ satisfies $\left.\alpha(i)=1\right\}$. Fix $i \in \mathbb{N}$. Clearly $q_{i}=P_{X(i)}^{(\vec{e})}$ satisfies $\pi\left(q_{i}\right)=\pi\left(p_{i}\right)$ and it is diagonalized by $\left(e_{n}\right)$.

Note that it is not true that any countable collection of commuting projections in $\mathcal{B}(H)$ is simultaneously diagonalizable (e.g., take $H=L^{2}([0,1])$ and the projections onto $L^{2}([0, q])$ for each $\left.q \in \mathbb{Q}\right)$.

Theorem 5.35 below was inspired by [41]. In this paper Luzin proved the existence of an uncountable almost disjoint family $\left\{X_{\xi}: \xi<\omega_{1}\right\}$ of subsets of $\mathbb{N}$ with the property that for every $\mathcal{Z} \subseteq \omega_{1}$ such that both $\mathcal{Z}$ and $\omega_{1} \backslash \mathcal{Z}$ are uncountable the families $\left\{X_{\xi}: \xi \in \overline{\mathcal{Z}}\right\}$ and $\left\{X_{\xi}: \xi \in \omega_{1} \backslash Z\right\}$ cannot be separated, in the sense that there is no $Y \subseteq \mathbb{N}$ such that $X_{\xi} \backslash Y$ is finite for all $\xi \in \mathcal{Z}$ and $X_{\xi} \cap Y$ is finite for all $\xi \in \omega_{1} \backslash \mathcal{Z}$ This family is one of the instances of incompactness of $\omega_{1}$ that are provable in ZFC, along with Hausdorff gaps, special Aronszajn trees, or nontrivial coherent families of partial functions.

Theorem 5.35 (Farah, $2006[22])$. There is a collection of $\aleph_{1}$ commuting projections in $\mathcal{C}(H)$ that cannot be lifted to simultaneous diagonalizable projections in $\mathcal{B}(H)$.

Proof. Construct $p_{\xi}\left(\xi<\omega_{1}\right)$ in $\mathcal{P}(\mathcal{B}(H))$ so that for $\xi \neq \eta$ (using the standard notation for the commutator of $a$ and $b,[a, b]=a b-b a)$ :

(1) $p_{\xi} p_{\eta}$ is compact, and

(2) $\left\|\left[p_{\xi}, p_{\eta}\right]\right\|>1 / 4$.

Such a family can easily be constructed by repeatedly applying Lemma 5.34.

If there are lifts $P_{X_{\xi}}^{(\vec{e})}$ of $\pi\left(p_{\xi}\right)$ that are all diagonalized by a basis $\left(e_{n}\right)$, let $d_{\xi}=p_{\xi}-P_{X(\xi)}^{(\vec{e})}$. Write $r_{n}=P_{\{0,1, \ldots, n-1\}}^{(\vec{e})}$, so $a$ is compact if and only if $\lim _{n}\left\|a\left(I-r_{n}\right)\right\|=0$. By hypothesis, each $d_{\xi}$ is compact, so fix $\bar{n}$ such that $S=\left\{\xi:\left\|d_{\xi}\left(I-r_{\bar{n}}\right)\right\|<1 / 8\right\}$ is uncountable. Since the range of $I-r_{\bar{n}}$ is separable, there are distinct $\xi, \eta \in S$ such that $\left\|\left(d_{\xi}-d_{\eta}\right) r_{\bar{n}}\right\|<1 / 8$. But then we can compute that

$$
\left\|\left[p_{\xi}, p_{\eta}\right]\right\| \leq\left\|\left[P_{X(\xi)}, P_{X(\eta)}\right]\right\|+1 / 8=1 / 4,
$$

a contradiction.

In the early draft of this paper it was conjectured that the projections constructed in Theorem 5.35 cannot be lifted to simultaneously commuting projections, and that in particular, $\mathfrak{l}=\aleph_{1}$. This conjecture was confirmed by Tristan Bice in [11].

5.5. Maximal chains of projections in the Calkin algebra. A problem closely related to cardinal invariants is the description of isomorphism classes of maximal chains in $\mathcal{P}(\mathbb{N}) /$ Fin and $\mathcal{P}(\mathcal{C}(H))$. The structure $(\mathcal{P}(\mathbb{N}) /$ Fin, $\leq)$ is $\aleph_{1}$-saturated, in the model-theoretic sense: every consistent type over a countable set is realized in the structure (this was first noticed by Hadwin 
in [34]). Therefore under $\mathrm{CH}$ a back-and-forth argument shows that all maximal chains are order-isomorphic. Countable saturatedness of quotients $\mathcal{P}(\mathbb{N}) / J$, for analytic ideals $J$, was well-studied. For example, by a result of Just and Krawczyk the quotient over every $F_{\sigma}$ ideal that includes Fin is countably saturated. Also, there are arbitrarily complex Borel ideals with countably saturated quotients. On the other hand, many well-studied $F_{\sigma \delta}$ ideals, for example the ideal $\mathcal{Z}_{0}$ of asymptotic density zero sets, don't have countably saturated quotients (see [20] and references thereof).

Theorem 5.36 (Hadwin, 1998 [34]). CH implies that any two maximal chains in $\mathcal{P}(\mathcal{C}(H))$ are order-isomorphic.

Proof. One can show that $\mathcal{P}(\mathcal{C}(H))$ has a similar saturation property and then use the same back-and-forth argument.

Conjecture 5.37 (Hadwin, 1998 [34]). CH is equivalent to "any two maximal chains in $\mathcal{P}(\mathcal{C}(H)$ ) are order-isomorphic".

This conjecture seems unlikely and the analogous statement for $\mathcal{P}(\mathbb{N}) /$ Fin is not true.

Theorem 5.38 (essentially Shelah-Steprāns). There is a model of $\neg \mathrm{CH}$ in which all maximal chains in $\mathcal{P}(\mathbb{N}) /$ Fin are isomorphic.

Proof. Add $\aleph_{2}$ Cohen reals to a model of $\mathrm{CH}$. We can then build up an isomorphism between any two maximal chains in the generic model in essentially the same way as a nontrivial automorphism of $\mathcal{P}(\mathbb{N}) /$ Fin is built up in [51].

The above proof cannot be straighforwardly adapted to the case of $\mathcal{P}(\mathcal{C}(H))$.

By forcing towers in $\mathcal{P}(\mathbb{N}) /$ Fin of different cofinalities, one can construct maximal chains in $(\mathcal{P}(\mathbb{N}) \backslash\{\mathbb{N}\}) /$ Fin of different cofinalities (in particular, they are non-isomorphic). The same thing works for $\mathcal{P}(\mathcal{C}(H)) \backslash\{\pi(I)\}$.

Theorem 5.39 (Wofsey, 2006 [59]). There is a forcing extension in which there are maximal chains in $\mathcal{P}(\mathcal{C}(H)) \backslash\{\pi(I)\}$ of different cofinalities (and $\left.2^{\aleph_{0}}=\aleph_{2}\right)$.

Idea of the proof. A standard forcing that adds maximal chains of different cofinalities to $\mathcal{P}(\mathbb{N}) /$ Fin works.

\section{More on PuRE States}

Recall that a state of a $\mathrm{C}^{*}$-algebra is pure if it cannot be written as a nontrivial linear combination of two distinct nonzero states (§4.1). We now look at some set-theoretic problems concerning pure states on $\mathrm{C}^{*}$-algebras.

Lemma 6.1. If $B$ is abelian and $A$ is a unital subalgebra of $B$ then any pure state of $B$ restricts to a pure state of $A$

Proof. A state on either algebra is pure if and only if it is multiplicative. It follows that the restriction of a pure state is pure. 
However, in general the restriction of a pure state to a unital subalgebra need not be pure.

Example 6.2. If $\omega_{\xi}$ is a vector state of $\mathcal{B}(H)$ and $\mathcal{A}$ is the atomic masa diagonalized by a basis $\left(e_{n}\right)$, then $\omega_{\xi}\left\lceil\mathcal{A}\right.$ is pure if and only if $\left|\left(\xi \mid e_{n}\right)\right|=1$ for some $n$. Indeed, $\mathcal{A}$ is isomorphic to $\ell^{\infty}$, which is in turn isomorphic to $C(\beta \mathbb{N})$. Therefore (cf. Exercise 4.16) a state of $\mathcal{A}$ is pure if and only if it is the evaluation functional at some point of $\beta \mathbb{N}$, or equivalently, if it is a limit of the vector states $\omega_{e_{n}}$ under an ultrafilter (such states reoccur in Example 6.31 below).

Lemma 6.3. If $A$ is an abelian $C^{*}$-algebra generated by its projections than a state $\phi$ of $A$ is pure if and only if $\phi(p) \in\{0,1\}$ for every projection $p$ in $A$.

Proof. Let us first consider the case when $A$ is unital. By the GelfandNamark theorem we may assume $A$ is $C(X)$ for a compact Hausdorff space $X$. By Exercise 4.16 a state $\phi$ of $C(X)$ is pure if and only if there is $x \in X$ such that $\phi(f)=f(x)$ for all $f$. Such a state clearly satisfies $\phi(p) \in\{0,1\}$ for each projection $p$ in $C(X)$.

If $\phi(p) \in\{0,1\}$ for every projection $p$, then $\mathcal{F}=\{p: \phi(p)=1\}$ is a filter such that for every $p$ either $p$ or $I-p$ is in $\mathcal{F}$. (Here $\mathcal{F}$ is a 'conventional' filter, not to be confused with quantum filters introduced after Lemma 6.41.) By our assumption, $X$ is zero-dimensional (cf. Exercise 5.11). Therefore $\mathcal{F}$ converges to a point $x$. We claim that $\phi(f)=f(x)$ for all $f \in C(X)$. Pick $f \in C(X)$ and $\epsilon>0$. Let $U \subseteq X$ be a clopen neighborhood of $x$ such that $|f(y)-f(x)|<\epsilon$ for all $y \in U$, and let $p$ be the projection corresponding to the characteristic function of $U$. Then $\phi(p)=1$ and by Lemma 4.8 we have $\phi(f)=\phi(p f p)$. On the other hand, with $\lambda=f(x)$ we have $\|p f p-\lambda p\|<\epsilon$, hence $|\phi(f)-\lambda|<\epsilon$. Since $\epsilon>0$ was arbitrary we conclude that $\phi(f)=\lambda=f(x)$.

If $A$ is not unital, then $A$ is isomorphic to $C_{0}(X)$ for a locally compact Hausdorff space $X$. Consider it as a subalgebra of $C(\beta X)$ and use an argument similar to the above.

Proposition 6.4. Let $B$ be a unital abelian $C^{*}$-algebra and $A \subseteq B$ be a unital subalgebra. If every pure state of $A$ extends to a unique pure state of $B$, then $A=B$.

Proof. We have $B=C(X)$, where $X$ is the space of pure states on $B$. Since $B$ is abelian, every point of $X$ gives a pure state on $A$. We claim that $A$ separates points of $X$ (cf. Exercise 3.11). Assume the contrary and let $x \neq y$ be points of $X$ such that $f(x)=f(y)$ for all $f \in A$. Then $f \mapsto f(x)$ is a pure state of $A$ that has two distinct extensions to a pure state of $B$, contradicting our assumption. By Stone-Weierstrass we have $A=C(X)$.

Without the assumption that $B$ is abelian the conclusion of Proposition 6.4 is no longer true. Let $B=M_{2}$ and let $A$ be its standard masa- the limit of algebras of diagonal matrices. Then $A$ is isomorphic to $C\left(2^{\mathbb{N}}\right)$ and 
each pure state $\phi$ of $A$ is an evaluation function at some $x \in 2^{\mathbb{N}}$. Assume $\psi$ is a state extension of $\phi$ to $M_{2^{\infty}}$. In each $M_{2^{n}}(\mathbb{C})$ there is a 1-dimensional projection $p_{n}$ such that $\phi\left(p_{n}\right)=1$, and therefore Lemma 4.8 implies that for all $a \in M_{2^{n}}(\mathbb{C})$ we have $\phi(a)=\phi\left(p_{n} a p_{n}\right)=$ the diagonal entry of the $2^{n} \times 2^{n}$ matrix $p_{n} a p_{n}$ determined by $p_{n}$. Since $\bigcup_{n} M_{2^{n}}(\mathbb{C})$ is dense in $M_{2^{\infty}}$, state $\psi$ is uniquely determined by $\phi$.

If $A \subseteq B$ are $\mathrm{C}^{*}$-algebras we say that $A$ separates pure states of $B$ if for all pure states $\psi \neq \phi$ of $B$ there is $a \in A$ such that $\phi(a) \neq \psi(a)$.

Exercise 6.5. Give an example of a $C^{*}$-algebra $B$ and its unital subalgebra $A$ such that $A$ separates pure states of $B$ but every pure state of $A$ has a unique extension to a state of $B$. (Hint: See Exercise 6.25.)

Problem 6.6 (Noncommutative Stone-Weierstrass problem). Assume $A$ is a unital subalgebra of $B$ and $A$ separates $\mathbb{P}(B) \cup\{0\}$. Does this necessarily imply $A=B$ ?

For more on this problem see e.g., [49].

Exercise 6.7. Prove that for an irreducible representation $\pi: A \rightarrow \mathcal{B}(H)$ we have $\pi[A] \supseteq \mathcal{K}(H)$ if and only if $\pi[A] \cap \mathcal{K}(H) \neq\{0\}$.

Definition 6.8 (Kaplansky). A $\mathrm{C}^{*}$-algebra $A$ is of type $I$ if for every irreducible representation $\pi: A \rightarrow \mathcal{B}(H)$ we have $\pi[A] \supseteq \mathcal{K}(H)$.

Type I $\mathrm{C}^{*}$-algebras are also known as GCR, postliminal, postliminary, or smooth. Here GCR stands for 'Generalized CCR' where CCR stands for 'completely continuous representation'; 'completely continuous operators' is an old-fashioned term for compact operators. See $[44, \S 6.2 .13]$ for an amusing explanation of the terminology (cf. footnote in $\S 5.1$ ). Type I C*algebras should not be confused with type I von Neumann algebras: $\mathcal{B}(H)$ is a type $\mathrm{I}$ von Neumann algebra but is not a type $\mathrm{I} \mathrm{C}^{*}$-algebra.

Definition 6.9. A $\mathrm{C}^{*}$-algebra is simple if and only if it has no nontrivial (closed two-sided) ideals.

Recall that the pure states of a $\mathrm{C}^{*}$-algebra correspond to its irreducible representations (Lemma 4.14) and that pure states are equivalent if and only if the corresponding irreducible representations are equivalent (Proposition 4.23).

Lemma 6.10. If a type $I C^{*}$-algebra has only one pure state up to equivalence then it is isomorphic to $\mathcal{K}(H)$ for some $H$.

Proof. Assume $A$ is of type I and all of its pure states are equivalent. It is not difficult to see that $A$ has to be simple. Therefore any irreducible representation is an isomorphism and therefore $\pi[A]=\mathcal{K}(H)$.

The converse of Lemma 6.10 is a theorem of Naimark (Theorem 6.14). $\mathrm{C}^{*}$-algebras that are not type I are called non-type I or antiliminary (cf. discussion of this terminology in the introduction to [8]). Theorem 6.11 is 
the key part of Glimm's characterization of type I C*-algebras ([31], see also [44, Theorem 6.8.7]). Its proof contains a germ of what became known as the Glimm-Effros Dichotomy ([35]).

Theorem 6.11 (Glimm). If $A$ is a non-type-I $C^{*}$-algebra then there is a subalgebra $B \subseteq A$ that has a quotient isomorphic to $M_{2}$.

Proof. See $[44, \S 6.8]$.

The following straightforward calculation will be used in Corollary 6.13.

Lemma 6.12. Assume $\varphi$ is a state of $A$ and $u$ and $v$ are unitaries in $A$ such that $\|u-v\|<\epsilon$. Then $\|\varphi \circ \operatorname{Ad} u-\varphi \circ \operatorname{Ad} v\|<2 \epsilon$.

Proof. It suffices to consider the case when $v=I$ and $\|u-I\|<\epsilon$. Then for $a \in A$ we have $\left\|a-u a u^{*}\right\|=\|a u-u a\| \leq\|a u-a\|+\|a-u a\|<2 \epsilon\|a\|$. Therefore we have $\left\|\varphi(a)-\varphi\left(u a u^{*}\right)\right\| \leq\left\|\varphi\left(a-u a u^{*}\right)\right\|<2 \epsilon\|a\|$ for all $a \in A$ and $\|\varphi-\operatorname{Ad} u \varphi\|<2 \epsilon$ follows.

Corollary 6.13 (Akemann-Weaver, 2002 [1]). If $A$ is non-type-I and has a dense subset of cardinality $<2^{\aleph_{0}}$, then $A$ has nonequivalent pure states.

Proof. By Glimm's Theorem, a quotient of a subalgebra of $A$ is isomorphic to $M_{2}$, and the pure states $\varphi_{f}$ on $M_{2}$ then lift and extend to pure states $\psi_{f}$ of $A$. Furthermore, if $f \neq g$ then $\left\|\psi_{f}-\psi_{g}\right\|=2$, since the same is true of $\varphi_{f}$ and $\varphi_{g}$. In particular, if $\psi$ is any pure state on $A$, then by Lemma 6.12 the unitaries that turn $\psi$ into $\psi_{f}$ must be far apart (distance $\geq 1$ ) from unitaries that turn $\psi$ into $\psi_{g}$. Since $A$ does not have a subset of cardinality $2^{\aleph_{0}}$ such that any two points are far apart from each other, $\psi$ cannot be equivalent to every $\psi_{f}$.

6.1. Naimark's theorem and Naimark's problem. The starting point of this subsection is the following converse of Lemma 6.10.

Theorem 6.14 (Naimark, 1948). Any two pure states on $\mathcal{K}(H)$ are equivalent, for any (not necessarily separable) Hilbert space $H$.

We shall sketch a proof of this theorem later on.

Question 6.15 (Naimark, 1951). If all pure states on a $C^{*}$-algebra $A$ are equivalent, is $A$ isomorphic to $\mathcal{K}(H)$ for some Hilbert space $H$ ?

Note that by Lemma 6.10 and Corollary 6.13 , any counterexample to this must be non-type I and have no dense subset of cardinality $<2^{\aleph_{0}}$. A similar argument shows that a counterexample cannot be a subalgebra of $\mathcal{B}(H)$ for a Hilbert space with a dense subset of cardinality $<2^{\aleph_{0}}$.

The proof of Naimark's theorem will require some terminology. Recall that a vector state on $\mathcal{B}(H)$ corresponding to a unit vector $\eta$ is defined by $\omega_{\eta}(a)=(a(\eta), \eta)$. 
Definition 6.16. An operator $a \in \mathcal{B}(H)$ is a trace class operator if for some orthogonal basis $E$ of $H$ we have $\sum_{e \in E}(|a| e, e)<\infty$. For a trace class operator $a$ define its trace as

$$
\operatorname{tr}(a)=\sum_{e \in E}(a e, e) .
$$

Exercise 6.17. Prove the following.

(1) Trace class operators form an ideal in $\mathcal{B}(H)$ that is not norm-closed. (Hint: See [45].)

(2) $\operatorname{tr}(a b)=\operatorname{tr}(b a)$ for any trace class operator $a$ and any operator $b$. In particular, this sum does not depend on the choice of the orthonormal basis.

(Hint: This is similar to the finite-dimensional case.)

(3) Every trace class operator is compact.

(Hint: It can be approximated by finite rank operators. by

For unit vectors $\eta_{1}$ and $\eta_{2}$ in $H$ define a rank one operator $b_{\eta_{1}, \eta_{2}}: H \rightarrow H$

$$
b_{\eta_{1}, \eta_{2}}(\xi)=\left(\xi, \eta_{2}\right) \eta_{1}
$$

This is a composition of the projection to $\mathbb{C} \eta_{2}$ with the partial isometry sending $\eta_{2}$ to $\eta_{1}$.

Lemma 6.18. Given a functional $\phi$ in the dual of $\mathcal{K}(H)$ there is a trace class operator $u$ such that $\phi(a)=\operatorname{tr}(u a)$ for all $a \in \mathcal{K}(H)$. If $\phi \geq 0$ then $u \geq 0$.

Proof. For the existence, see e.g., [45, Theorem 3.4.13]. To see $u$ is positive, pick $\eta \in H$. Then $u b_{\eta, \eta}(\xi)=u((\xi, \eta) \eta)=(\xi, \eta) u(\eta)=b_{u(\eta), \eta}(\xi)$. Therefore

$$
\begin{aligned}
0 \leq \phi\left(b_{\eta, \eta}\right)=\operatorname{tr}\left(u b_{\eta, \eta}\right)= & \operatorname{tr}\left(b_{u(\eta), \eta}\right) \\
& =\sum_{e \in E}\left(b_{u(\eta), \eta}(e), e\right)=\sum_{e \in E}\left(u b_{\eta, \eta} e, e\right)=(u(\eta), \eta) .
\end{aligned}
$$

(In the last equality we change the basis to $E^{\prime}$ so that $\eta \in E^{\prime}$.)

Proposition 6.19. Every pure state $\phi$ of $\mathcal{K}(H)$ is equal to the restriction of some vector state to $\mathcal{K}(H)$.

Proof. By Lemma 6.18 we have a trace class operator $u$ such that $\phi(a)=$ $\operatorname{tr}(u a)$ for all $a \in \mathcal{K}(H)$. Since $u$ is a positive compact operator, it is by the Spectral Theorem diagonalizable so we can write $u=\sum_{e \in E} \lambda_{e} e^{*}$ with the appropriate choice of the basis $E$. Thus $\phi(a)=\operatorname{tr}(u a)=\operatorname{tr}(a u)=$ $\sum_{e \in E}($ aue,$e)=\sum_{e \in E} \lambda_{e}(a e, e) \geq \lambda_{e_{0}}\left(a e_{0}, e_{0}\right)$, for any $e_{0} \in E$. Since $\phi$ is a pure state, for each $e \in E$ there is $t_{e} \in[0,1]$ such that $t_{e} \phi(a)=\lambda_{e_{0}}\left(a e_{0}, e_{0}\right)$. Thus exactly one $t_{e}=t_{e_{0}}$ is nonzero, and $a \mapsto \lambda_{e_{0}}\left(a e_{0}, e_{0}\right)$.

Proof of Theorem 6.14. If $\xi$ and $\eta$ are unit vectors in $H$, then the corresponding vector states $\omega_{\xi}$ and $\omega_{\eta}$ are clearly equivalent, via any unitary that sends $\xi$ to $\eta$. Hence the conclusion follows from Proposition 6.19 below. 
6.2. A counterexample to Naimark's problem from $\diamond$. We shall now sketch a recent result of Akemann and Weaver, giving a consistent counterexample to Naimark's problem. One of the most interesting set-theoretic problems about $\mathrm{C}^{*}$-algebras is whether a positive solution to Naimark's problem is consistent with ZFC. A positive answer would open a possibility of having an interesting representation theory for not necessarily separable $\mathrm{C}^{*}$-algebras (see the introduction to [1]). The following lemma is based on recent work of Kishimoto-Ozawa-Sakai and Futamura-Kataoka-Kishimoto.

Lemma 6.20 (Akemann-Weaver, 2004 [1]). Let $A$ be a simple separable unital $C^{*}$-algebra and let $\varphi$ and $\psi$ be pure states on $A$. Then there is a simple separable unital $B \supseteq A$ such that

(1) $\varphi$ and $\psi$ extend to states $\varphi^{\prime}, \psi^{\prime}$ on $B$ in a unique way.

(2) $\varphi^{\prime}$ and $\psi^{\prime}$ are equivalent.

Proof. Omitted.

It is not known whether this lemma remains true when the separability assumption is dropped. However, Kishimoto-Ozawa-Sakai proved that their result used in the proof of Lemma 6.20 fails for nonseparable algebras. A very simple example was given in [25].

We shall now briefly describe Jensen's $\diamond$ principle, and the set-theoretically informed readers may want to skip ahead to Theorem 6.22. Recall that a subset $C$ of $\omega_{1}$ is closed if for every countable $A \subseteq C$ we have that $\sup A \in C$. It is unbounded if it $\sup C=\omega_{1}$. By $\diamond$ we denote Jensen's diamond principle on $\omega_{1}$. One of its equivalent reformulations states that there are functions $h_{\alpha}: \alpha \rightarrow \omega_{1}$, for $\alpha<\omega_{1}$, such that for every $g: \omega_{1} \rightarrow \omega_{1}$, the set $\left\{\alpha: g\left\lceil\alpha=h_{\alpha}\right\}\right.$ is stationary.

There are several revealing reformulations of $\diamond$ (see [40, Chapter II]), and the following one was suggested by Weaver.

Exercise 6.21. Consider $T=\omega_{1}^{<\omega_{1}}$ as a tree with respect to the endextension ordering. Show that $\diamond$ is equivalent to the assertion that there is $t_{\alpha}$ in $T$ of length $\alpha$ such that for every $\omega_{1}$-branch $b$ of $T$ the set of all $\alpha$ such that $b\left\lceil\alpha=t_{\alpha}\right.$ is stationary.

Theorem 6.22 (Akemann-Weaver, 2004 [1]). Assume $\diamond$. Then there is a $C^{*}$-algebra $A$, all of whose pure states are equivalent, which is not isomorphic to $\mathcal{K}(H)$ for any $H$.

Proof. We construct an increasing chain of simple separable unital $\mathrm{C}^{*}$-algebras $A_{\alpha}\left(\alpha \leq \omega_{1}\right)$. We also construct pure states $\psi_{\alpha}$ on $A_{\alpha}$ such that for $\alpha<\beta, \psi_{\beta}\left\lceil A_{\alpha}=\psi_{\alpha}\right.$. For each $A_{\alpha}$, let $\left\{\varphi_{\alpha}^{\gamma}\right\}_{\gamma<\omega_{1}}$ enumerate all of its pure states.

If $\alpha$ is limit, we let $A_{\alpha}=\lim _{\beta \rightarrow \alpha} A_{\beta}$ and $\psi_{\alpha}=\lim _{\longrightarrow} \psi_{\beta}$.

Let us consider the successor case, when $A_{\alpha}$ is defined and we want to define $A_{\alpha+1}$. Suppose there is $\varphi \in \mathbb{P}\left(A_{\alpha}\right)$ such that $\varphi\left\lceil A_{\beta}=\varphi_{\beta}^{h_{\alpha}(\beta)}\right.$ for all 
$\beta<\alpha$ (if no such $\varphi$ exists, let $A_{\alpha+1}=A_{\alpha}$ ). Note that $\bigcup_{\beta<\alpha} A_{\beta}$ is dense in $A_{\alpha}$ since $\alpha$ is limit, so there is at most one such $\varphi$. By Lemma 6.20, let $A_{\alpha+1}$ be such that $\psi_{\alpha}$ and $\varphi$ have unique extensions to $A_{\alpha+1}$ that are equivalent, and let $\psi_{\alpha+1}$ be the unique extension of $\psi_{\alpha}$.

Let $A=A_{\omega_{1}}$ and $\psi=\psi_{\omega_{1}}$. Then $A$ is unital and infinite-dimensional, so $A$ is not isomorphic to any $\mathcal{K}(H)$. Let $\varphi$ be any pure state on $A$; we will show that $\varphi$ is equivalent to $\psi$, so that $A$ has only one pure state up to equivalence.

Claim 6.23. $S=\left\{\alpha: \varphi \mid A_{\alpha}\right.$ is pure on $\left.A_{\alpha}\right\}$ contains a club.

Proof. For $x \in A$ and $m \in \mathbb{N}$ the set

$$
\begin{aligned}
T_{m, x}=\left\{\alpha: x \in A_{\alpha} \text { and }\left(\exists \psi_{1}, \psi_{2} \in \mathbb{S}\left(A_{\alpha}\right)\right)\right. & \\
& \left.\varphi \mid A_{\alpha}=\frac{\psi_{1}+\psi_{2}}{2} \text { and }\left|\varphi(x)-\psi_{1}(x)\right| \geq \frac{1}{m}\right\}
\end{aligned}
$$

is bounded in $\omega_{1}$. Indeed, if it were unbounded, we could take a limit of such $\psi_{i}$ (with respect to an ultrafilter) to obtain states $\psi_{i}$ on $A$ such that $\varphi=\frac{\psi_{1}+\psi_{2}}{2}$ but such that $\left|\varphi(x)-\psi_{1}(x)\right| \geq \frac{1}{m}$, contradicting purity of $\varphi$. Since each $A_{\alpha}$ is separable, we can take a suitable diagonal intersection of the $T_{m, x}$ over all $m$ and all $x$ in a dense subset of $A$ to obtain a club contained in $S$.

Now let $h: S \rightarrow \omega_{1}$ be such that $\varphi\left\lceil A_{\alpha}=\varphi_{\alpha}^{h(\alpha)}\right.$ for all $\alpha \in S$. Since $S$ contains a club, there is some limit ordinal $\alpha$ such that $h\left\lceil\alpha=h_{\alpha}\right.$. Then by construction, $\varphi\left\lceil A_{\alpha+1}\right.$ is equivalent to $\psi_{\alpha+1}$; say $\varphi\left\lceil A_{\alpha+1}=u \psi_{\alpha+1} u^{*}\right.$ for a unitary $u$. For each $\beta \geq \alpha, \psi_{\beta}$ extends uniquely to $\psi_{\beta+1}$, so by induction we obtain that $\psi$ is the unique extension of $\psi_{\alpha+1}$ to $A$. Since $\varphi\left\lceil A_{\alpha+1}\right.$ is equivalent to $\psi_{\alpha+1}$, it also has a unique extension to $A$, which must be $\varphi$. But $u \psi u^{*}$ is an extension of $\varphi\left\lceil A_{\alpha+1}\right.$, so $\varphi=u \psi u^{*}$ and is equivalent to $\psi$.

6.3. Extending pure states on masas. By Exercise 4.16, a state on an abelian $\mathrm{C}^{*}$-algebra is pure if and only if it is multiplicative, i.e., a ${ }^{*}$-homomorphism. If the algebra is generated by projections then this is equivalent to asserting that $\phi(p) \in\{0,1\}$ for every projection $p$ (Lemma 6.3).

Definition 6.24. A masa in a $\mathrm{C}^{*}$-algebra $A$ has the extension property (EP) if each of its pure states extends uniquely to a pure state on $A$.

If $\mathcal{A} \subseteq \mathcal{B}(H)$ is a masa and $\phi$ is a vector state on $\mathcal{A}$ then $\phi$ extends uniquely to a pure state of $\mathcal{B}(H)$. This is essentially an easy consequence of Lemma 4.8. By Theorem 4.21 all non-vector pure states are singular and thus define pure states on $\mathcal{C}(H)$. These two observations together imply that a masa $\mathcal{A} \subset \mathcal{B}(H)$ has the EP if and only if $\pi[\mathcal{A}] \subset \mathcal{C}(H)$ has the EP

Exercise 6.25. Let $\mathcal{A}$ be the CAR algebra and let $\mathcal{D}$ be the masa generated by diagonal matrices (cf. Exercise 5.22). Show that $\mathcal{D}$ has the extension 
property. (Hint: Do the finite-dimensional case first. That is, show that the masa consisting of diagonal matrices in $M_{n}(\mathbb{C})$ has the extension property. See also Exercise 4.11.)

Theorem 6.26 (Kadison-Singer, 1959, [38]). Atomless masas in $\mathcal{B}(H)$ do not have the EP.

Proof. Omitted.

Theorem 6.27 (Anderson, $1978[5])$. CH implies there is a masa in $\mathcal{C}(H)$ that has the EP.

Proof. Omitted.

Note that Anderson's theorem does not give a masa on $\mathcal{B}(H)$ with the $\mathrm{EP}$, since his masa on $\mathcal{C}(H)$ does not lift to a masa on $\mathcal{B}(H)$. The following is a famous open problem (compare with Problem 6.6).

Problem 6.28 (Kadison-Singer, 1959 [38]). Do atomic masas of $\mathcal{B}(H)$ have the EP?

This is known to be equivalent to an arithmetic statement (i.e., a statement all of whose quantifiers range over natural numbers). As such, it is absolute between transitive models of ZFC and its solution is thus highly unlikely to involve set theory. For more on this problem see [15] and [56]. However, there are related questions that seem more set-theoretic. For example, consider the following conjecture:

Conjecture 6.29 (Kadison-Singer, 1959 [38]). For every pure state $\varphi$ of $\mathcal{B}(H)$ there is a masa $\mathcal{A}$ such that $\varphi \mid \mathcal{A}$ is multiplicative (i.e., pure).

We could also make the following stronger conjecture:

Conjecture 6.30. For every pure state $\varphi$ of $\mathcal{B}(H)$ there is an atomic masa $\mathcal{A}$ such that $\varphi\lceil\mathcal{A}$ is multiplicative.

Example 6.31. Let $\mathcal{U}$ be an ultrafilter on $\mathbb{N}$ and $\left(e_{n}\right)$ be an orthonormal basis for $H$. Then

$$
\varphi_{\mathcal{U}}^{(\vec{e})}(a)=\lim _{n \rightarrow \mathcal{U}}\left(a e_{n} \mid e_{n}\right)
$$

is a state on $\mathcal{B}(H)$. It is singular if and only if $\mathcal{U}$ is nonprincipal (if $\{n\} \in \mathcal{U}$, then $\left.\varphi_{\mathcal{U}}^{(\vec{e})}=\omega_{e_{n}}\right)$.

We say a state of the form $\varphi_{\mathcal{U}}^{(\overrightarrow{\mathcal{U}})}$ for some basis $\left(e_{n}\right)$ and some ultrafilter $\mathcal{U}$ is diagonalizable. As noted in Example 6.2, the restriction of a diagonalizable state to the corresponding atomic masa is a pure state of the masa, and every pure state of an atomic masa is of this form.

Theorem 6.32 (Anderson, 1979 [7]). Diagonalizable states are pure.

Proof. Omitted.

Conjecture 6.33 (Anderson, $1981[4])$. Every pure state on $\mathcal{B}(H)$ is diagonalizable. 
Proposition 6.34. If atomic masas do have the EP, then Anderson's conjecture is equivalent to Conjecture 6.30.

Proof. If atomic masas have the EP, a pure state on $\mathcal{B}(H)$ is determined by its restriction to any atomic masa on which it is multiplicative. Any multiplicative state on an atomic masa extends to a diagonalizable state, so this means that a pure state restricts to a multiplicative state if and only if it is diagonalizable.

We now prove an affirmative answer for a special case of the KadisonSinger problem. We say an ultrafilter $\mathcal{U}$ on $\mathbb{N}$ is a $Q$-point (sometimes called rare ultrafilter) if every partition of $\mathbb{N}$ into finite intervals has a transversal in $\mathcal{U}$. The existence of Q-points is known to be independent from ZFC, but what matters here is that many ultrafilters on $\mathbb{N}$ are not Q-points.

Fix a basis $\left(e_{n}\right)$ and let $\mathcal{A}$ denote the atomic masa of all operators diagonalized by it. In the following proof we write $P_{X}$ for $P_{X}^{(\vec{e})}$.

Theorem 6.35 (Reid, 1971 [47]). If $\mathcal{U}$ is a Q-point then the diagonal state $\varphi \mathcal{U}\lceil\mathcal{A}$ has a unique extension to a pure state of $\mathcal{B}(H)$.

Proof. Fix a pure state $\varphi$ on $\mathcal{B}(H)$ extending $\varphi_{\mathcal{U}}\lceil\mathcal{A}$ and let $a \in \mathcal{B}(H)$. Without a loss of generality $\mathcal{U}$ is nonprincipal so $\varphi$ is singular.

Choose finite intervals $\left(J_{i}\right)$ such that $\mathbb{N}=\bigcup_{n} J_{n}$ and

$$
\left\|P_{J_{m}} a P_{J_{n}}\right\|<2^{-m-n}
$$

whenever $|m-n| \geq 2$. This is possible by (2) and (3) of Example 3.1.4 since $a P_{J_{m}}$ and $P_{J_{m}} a$ are compact. (See [23, Lemma 1.2] for details.) Let $X \in \mathcal{U}$ be such that $X \cap\left(J_{2 i} \cup J_{2 i+1}\right)$ has a unique element, $n(i)$, for all $i$. Then for $Q_{i}=P_{\{n(i)\}}$ and $f_{i}=e_{n(i)}$ we have $\varphi\left(\sum_{i} Q_{i}\right)=1$ and

$$
Q a Q=\sum_{i} Q_{i} a \sum_{i} Q_{i}=\sum_{i} Q_{i} a Q_{i}+\sum_{i \neq j} Q_{i} a Q_{j} .
$$

The second sum is compact by our choice of $\left(J_{i}\right)$, and $Q_{i} a Q_{i}=\left(a f_{i} \mid f_{i}\right) Q_{i}$. Now as we make $X \in \mathcal{U}$ smaller and smaller, $\sum_{i \in X}\left(a e_{i} \mid e_{i}\right) P_{\{i\}}$ gets closer and closer to $\left(\lim _{i \rightarrow \mathcal{U}}\left(a e_{i} \mid e_{i}\right)\right) \sum P_{i}=\varphi_{\mathcal{U}}(a) \sum P_{i}$. Thus

$$
\lim _{X \rightarrow \mathcal{U}} \pi\left(P_{X} a P_{X}-\varphi_{\mathcal{U}}(a) P_{X}\right) \rightarrow 0 .
$$

Since $\varphi$ is singular and $\varphi\left(P_{X}\right)=\varphi_{\mathcal{U}}\left(P_{X}\right)=1$, by Lemma $4.8 \varphi(a)=$ $\varphi\left(P_{X} a P_{X}\right)=\varphi_{\mathcal{U}}(a)$. Since $a$ was arbitrary, $\varphi=\varphi \mathcal{U}$.

6.4. A pure state that is not multiplicative on any masa in $\mathcal{B}(H)$. The following result shows that Conjecture 6.30 is not true in all models of ZFC. The following theorem follows from a stronger result, Theorem 6.46, whose proof will be sketched below.

Theorem 6.36 (Akemann-Weaver, $2005[2]$ ). CH implies there is a pure state $\varphi$ on $\mathcal{B}(H)$ that is not multiplicative on any atomic masa. 
The basic idea of constructing such a pure state is to encode pure states as "quantum ultrafilters"; a pure state on the atomic masa $\ell^{\infty} \subset \mathcal{B}(H)$ is equivalent to an ultrafilter. By the following result, states on $\mathcal{B}(H)$ correspond to finitely additive maps from $\mathcal{P}(\mathcal{B}(H))$ into $[0,1]$.

Theorem 6.37 (Gleason). Assume $\mu: \mathcal{P}(\mathcal{B}(H)) \rightarrow[0,1]$ is such that $\mu(p+$ $q)=\mu(p)+\mu(q)$ whenever $p q=0$. Then there is a unique state on $\mathcal{B}(H)$ that extends $\mu$.

Proof. Omitted.

We need to go a little further and associate certain 'filters' of projections to pure states of $\mathcal{B}(H)$.

Definition 6.38. A family $\mathbb{F}$ of projections in a $\mathrm{C}^{*}$-algebra is a filter if

(1) For any $p, q \in \mathbb{F}$ there is $r \in \mathbb{F}$ such that $r \leq p$ and $r \leq q$.

(2) If $p \in \mathbb{F}$ and $r \geq p$ then $r \in \mathbb{F}$.

The filter generated by $\mathbb{X} \subseteq \mathcal{P}(A)$ is the intersection of all filters containing $\mathbb{X}$ (which may not actually be a filter in general if $\mathcal{P}(A)$ is not a lattice).

We say that a filter $\mathcal{F} \subset \mathcal{P}(\mathcal{C}(H))$ lifts if there is a commuting family $\mathbb{X} \subseteq \mathcal{P}(\mathcal{B}(H))$ that generates a filter $\mathbb{F}$ such that $\pi[\mathbb{F}]=\mathcal{F}$. Note that, unlike the case of quotient Boolean algebras, $\pi^{-1}[\mathcal{F}]$ itself is not a filter because there exist projections $p, q \in \mathcal{B}(H)$ such that $\pi(p)=\pi(q)$ but $p \wedge q=0$ (Lemma 5.25).

Question 6.39. Does every maximal filter $\mathcal{F}$ in $\mathcal{P}(\mathcal{C}(H))$ lift?

Maximal filters in $\mathcal{P}(\mathcal{C}(H))$ can have rather interesting properties, as the following result shows.

Theorem 6.40 (Anderson, $[6]$ ). There are a singular pure state $\varphi$ of $\mathcal{B}(H)$, an atomic masa $\mathcal{A}_{1}$, and an atomless masa $\mathcal{A}_{2}$ such that both $\varphi\left\lceil\mathcal{A}_{1}\right.$ and $\varphi\left\lceil\mathcal{A}_{2}\right.$ are multiplicative.

Proof. Omitted.

Lemma 6.41 (Weaver, 2007). For $\mathcal{F}$ in $\mathcal{P}(\mathcal{B}(H)$ ) the following are equivalent:

(A) $\left\|p_{1} p_{2} \cdots p_{n}\right\|=1$ for any $p_{1}, \cdots, p_{n} \in \mathcal{F}$ and $\mathcal{F}$ is maximal with respect to this property.

(B) For all $\epsilon>0$ and for all finite $F \subseteq \mathcal{F}$ there is a unit vector $\xi$ such that $\|p \xi\|>1-\epsilon$ for all $p \in F$.

Proof. Since $\left\|p_{1} p_{2} \cdots p_{n}\right\| \leq\left\|p_{1}\right\| \cdot\left\|p_{2}\right\| \cdot \ldots \cdot\left\|p_{n}\right\|=1$, clause (A) is equivalent to stating that for every $\epsilon>0$ there is a unit vector $\xi$ such that $\left\|p_{1} p_{2} \cdots p_{n} \xi\right\|>1-\epsilon$. The remaining calculations are left as an exercise to the reader. Keep in mind that, for a projection $p$, the value of $\|p \xi\|$ is close to $\|\xi\|$ if and only if $\|\xi-p \xi\|$ is close to 0 . 
We call an $\mathcal{F}$ satisfying the conditions of Lemma 6.41 a quantum filter. Such an $\mathcal{F}$ is a maximal quantum filter if it is not properly included in another quantum filter.

Theorem 6.42 (Farah-Weaver, 2007). Let $\mathcal{F} \subseteq \mathcal{P}(\mathcal{C}(H))$. Then the following are equivalent:

(1) $\mathcal{F}$ is a maximal quantum filter,

(2) $\mathcal{F}=\mathcal{F}_{\varphi}=\{p: \varphi(p)=1\}$ for some pure state $\varphi$.

Proof. $(1 \Rightarrow 2)$ : For a finite $F \subseteq \mathcal{F}$ and $\epsilon>0$ let

$$
X_{F, \epsilon}=\{\varphi \in \mathbb{S}(\mathcal{B}(H)): \varphi(p) \geq 1-\epsilon \text { for all } p \in F\} .
$$

If $\xi$ is as in (B) then $\omega_{\xi} \in X_{F, \epsilon}$.

Since $X_{F, \epsilon}$ is weak ${ }^{*}$-compact, $\bigcap_{(F, \epsilon)} X_{F, \epsilon} \neq \emptyset$, and any extreme point of the intersection is a pure state with the desired property. ${ }^{3}$

$(2 \Rightarrow 1)$. If $\varphi\left(p_{j}\right)=1$ for $j=1, \ldots, k$, then $\varphi\left(p_{1} p_{2} \ldots p_{k}\right)=1$ by Lemma 4.8 , hence $(\mathrm{A})$ holds. It is then not hard to show that $\mathcal{F}_{\varphi}$ also satisfies (B) and is maximal.

Lemma 6.43. Let $\mathcal{F}$ be a maximal quantum filter, let $\left(\xi_{n}\right)$ be an orthonormal basis, and let $\mathbb{N}=\bigcup_{j=1}^{n} A_{j}$ be a finite partition. If there is a $q \in \mathcal{F}$ such that $\left\|P_{A_{j}}^{(\vec{\xi})} q\right\|<1$ for all $j$, then $\mathcal{F}$ is not diagonalized by $\left(\xi_{n}\right)$. In other words, the corresponding pure state is not diagonalized by $\left(\xi_{n}\right)$.

Proof. Assume $\mathcal{F}$ is diagonalized by $\left(\xi_{n}\right)$ and let $\mathcal{U}$ be such that $\mathcal{F}=\varphi_{\mathcal{U}}^{(\vec{\xi})}$. Then $A_{j} \in \mathcal{U}$ for some $j$, but $\left\|P_{A_{j}}^{(\vec{\xi})} q\right\|<1$ for $q \in \mathcal{F}$, contradicting the assumption that $\mathcal{F}$ is a filter.

Lemma 6.44. Let $\left(e_{n}\right)$ and $\left(\xi_{n}\right)$ be orthonormal bases. Then there is a partition of $\mathbb{N}$ into finite intervals $\left(J_{n}\right)$ such that for all $k$,

$$
\xi_{k} \in \overline{\operatorname{span}}\left\{e_{i}: i \in J_{n} \cup J_{n+1}\right\}
$$

(modulo a small perturbation of $\xi_{k}$ ) for some $n=n(k)$.

Proof. Omitted.

For $\left(J_{n}\right)$ as in Lemma 6.44 let

$$
D_{\vec{J}}=\left\{q:\left\|P_{J_{n} \cup J_{n+1}}^{(\vec{e})} q\right\|<1 / 2 \text { for all } n\right\}
$$

Lemma 6.45. Each $D_{\vec{J}}$ is dense in $\mathcal{P}(\mathcal{C}(H))$, in the sense that for any noncompact $p \in \mathcal{P}(\mathcal{B}(H))$, there is a noncompact $q \leq p$ such that $q \in D_{\vec{J}}$.

Proof. Taking a basis for range $(p)$, we can thin out the basis and take appropriate linear combinations to find such a $q$.

\footnotetext{
${ }^{3}$ It can be proved, using a version of Kadison's Transitivity Theorem ([32]), that this intersection is actually a singleton.
} 
Recall that $\mathfrak{d}$ is the minimal cardinality of a cofinal subset of $\mathbb{N}^{\mathbb{N}}$ under the pointwise order, and we write $\mathfrak{t}^{*}$ for the minimal length of a maximal decreasing well-ordered chain in $\mathcal{P}(\mathcal{C}(H)) \backslash\{0\}$. In particular, $\mathrm{CH}$ (or MA) implies that $\mathfrak{d}=\mathfrak{t}^{*}=2^{\aleph_{0}}$.

Theorem 6.46 (Farah-Weaver). Assume $\mathfrak{d} \leq \mathfrak{t}^{*}{ }^{4}$ Then there exists a pure state on $\mathcal{B}(H)$ that is not diagonalized by any atomic masa.

Proof. We construct a corresponding maximal quantum filter. By the density of $D_{\vec{J}}$ and $\mathfrak{d} \leq \mathfrak{t}^{*}$, it is possible to construct a maximal quantum filter $\mathcal{F}$ such that $\mathcal{F} \cap D_{\vec{J}} \neq \emptyset$ for all $\vec{J}$. Given a basis $\left(\xi_{k}\right)$, pick $\left(J_{n}\right)$ such that $\xi_{k} \in J_{n(k)} \cup J_{n(k)+1}$ (modulo a small perturbation) for all $k$. Let $A_{i}=\{k \mid n(k)=i(\bmod 4)\}$ for $0 \leq i<4$. Then if $q \in \mathcal{F} \cap D_{\vec{J}},\left\|P_{A_{i}}^{(\vec{\xi})} q\right\|<1$ for each $i$. By Lemma $6.43, \mathcal{F}$ is not diagonalized by $\left(\xi_{n}\right)$.

\section{Automorphisms of the Calkin algebra}

We now investigate whether the Calkin algebra has nontrivial automorphisms. Since the Calkin algebra is a quantized version of the Cech-Stone compactification of the natural numbers, this question is analogous to the question of whether $\mathcal{P}(\mathbb{N}) /$ Fin has nontrivial automorphisms. This question first appeared in its dual, topological, form (see §5.0.1): does the Cech-Stone remainder $\beta \mathbb{N} \backslash \mathbb{N}$ of $\mathbb{N}$ have nontrivial autohomeomorphisms? Here we say that an autohomeomorphism $f$ is trivial if it has a continuous extension to a map $\bar{f}: \beta \mathbb{N} \rightarrow \beta \mathbb{N}$ (note that $\bar{f}$ is not necessarily an autohomeomorphism).

This question was consistently answered by W. Rudin, who proved that $\mathrm{CH}$ implies the existence of $2^{\mathfrak{c}}$ nontrivial autohomeomorphisms of $\beta \mathbb{N} \backslash \mathbb{N}$. Later Shelah $([50])$ constructed a forcing extension in which all automorphisms of $\mathcal{P}(\mathbb{N}) /$ Fin are trivial. Towards this end he has developed a sophisticated oracle chain condition forcing. It is interesting that Shelah's result came only a few years after the Brown-Douglas-Fillmore question, but it took almost thirty years before the connection was noticed by Weaver in [58].

Shelah's conclusion was later obtained from the Proper Forcing Axiom (Shelah-Steprāns) and from Todorcevic's Axiom (which is one of the two axioms known under the name of Open Coloring Axiom, OCA) and Martin's Axiom (Veličković). A number of rigidity results along the similar lines have been obtained since (see [21]).

We say an automorphism $\Phi$ of a $\mathrm{C}^{*}$-algebra is inner if $\Phi=\mathrm{Ad} u$ for some unitary $u$.

Example 7.1. If $A=C_{0}(X)$ is abelian then each automorphism is of the form

$$
f \mapsto f \circ \Psi
$$

\footnotetext{
${ }^{4}$ The sharpest hypothesis would be $\mathfrak{d}<$ "the Novák number of $\mathcal{P}(\mathcal{C}(H))$ ", if it makes sense.
} 
for an autohomeomorphism $\Psi$ of $X$. This automorphism is inner if and only if $\Psi$ is the identity since abelian structures cannot have nontrivial inner automorphisms. Thus abelian $\mathrm{C}^{*}$-algebras often have many outer automorphisms since every outer autohomeomorphism of the underlying compact Hausdorff space defines a (necessarily outer) automorphism of the algebra. However, there do exist (locally) compact Hausdorff spaces with no nontrivial autohomeomorphisms (see the introduction of [46]), so some nontrivial abelian $\mathrm{C}^{*}$-algebras have no outer automorphisms.

Proposition 7.2. All automorphisms of $\mathcal{B}(H)$ are inner.

Proof. Omitted, but not too different from the proof that each automorphism of $\mathcal{P}(\mathbb{N})$ is given by a permutation of $\mathbb{N}$.

Proposition 7.3. The CAR algebra $M_{2}=\bigotimes_{n} M_{2}(\mathbb{C})$ has outer automorphisms.

Proof. Let $\Phi=\bigotimes_{n} \operatorname{Ad}\left(\begin{array}{ll}0 & 1 \\ 1 & 0\end{array}\right)$. Then consider

$$
a_{n}=\bigotimes_{i<n}\left(\begin{array}{ll}
1 & 0 \\
0 & 1
\end{array}\right) \otimes\left(\begin{array}{cc}
1 & 0 \\
0 & -1
\end{array}\right)
$$

as an element of $M_{2^{n}}(\mathbb{C}) \subseteq M_{2^{\infty}}$. We have $\Phi\left(a_{n}\right)=-a_{n}$, and hence $\| \Phi\left(a_{n}\right)$ $a_{n} \|=2$. Since every element of $M_{2}$ can be approximated arbitrarily closely by an element of a large enough $M_{2^{n}}(\mathbb{C})$, for every $u \in M_{2^{\infty}}$ we have that $\lim _{n}\left\|(\operatorname{Ad} u)\left(a_{n}\right)-a_{n}\right\|=0$. This implies that for every inner automorphism Ad $u$ of $M_{2}$ we have $\lim _{n}\left\|(\operatorname{Ad} u)\left(a_{n}\right)-\Phi\left(a_{n}\right)\right\|=2$, and therefore $\Phi$ is outer.

Exercise 7.4. Assume $A$ is a $\mathrm{C}^{*}$-algebra that is not inner.

(1) Show that every automorphism of $A$ extends to the unique automorphism of its unitization $\tilde{A}$.

(2) Using (1) say that an automorphism of a non-unital algebra is outer if the corresponding automorphism of $\tilde{A}$ is outer. Show that for an infinite-dimensional $H$ the algebra $\mathcal{K}(H)$ has outer automorphisms.

The fact is that all infinite-dimensional simple separable algebras have outer automorphisms. We now turn to automorphisms of the Calkin algebra.

Theorem 7.5 (Brown-Douglas-Fillmore, $1977[14]$ ). Let $a, b \in \mathcal{B}(H)$ be normal. Then there is an automorphism of $\mathcal{C}(H)$ mapping $\pi(a)$ to $\pi(b)$ if and only if there is an inner automorphism of $\mathcal{C}(H)$ mapping $\pi(a)$ to $\pi(b)$.

Proof. Omitted

Question 7.6 (Brown-Douglas-Fillmore, 1977 [14, 1.6(ii)]). Is there an automorphism of $\mathcal{C}(H)$ that maps $\pi(S)$ to $\pi\left(S^{*}\right)$ ? More generally, are there $a, b \in \mathcal{C}(H)$ such that no inner automorphism maps a to $b$ but an outer automorphism does? 
Although unitaries in $\mathcal{C}(H)$ need not lift to unitaries in $\mathcal{B}(H)$, they do lift to "almost unitaries" (i.e., isomorphisms between finite codimension subspaces of $H)$. In particular, if we let $S$ denote the unilateral shift, one can show that no inner automorphism of $\mathcal{C}(H)$ sends $\pi(S)$ to $\pi\left(S^{*}\right)$. This is because conjugation by a partial isometry preserves the Fredholm index, and the Fredholm index of $S$ is -1 while the Fredholm index of $S^{*}$ is 1 .

A more basic question than Question 7.6, also asked by Brown, Douglas and Fillmore ([14, 1.6(ii)]), is whether outer automorphisms exist at all. The obvious approach to construct an outer automorphism would be to simply take a nontrivial automorphism of $\mathcal{P}(\mathbb{N}) /$ Fin $\subset \mathcal{P}(\mathcal{C}(H))$ and try to extend it to an automorphism of all of $\mathcal{C}(H)$. Unfortunately, this does not work, by the following consequence of a result of Alperin-Covington-Macpherson [3].

Proposition 7.7. No nontrivial automorphism of $\mathcal{P}(\mathbb{N}) /$ Fin extends to an automorphism of $\mathcal{C}(H)$.

Proof. Recall that $S_{\infty}$ is the group of all permutations of $\mathbb{N}$ and let $\operatorname{FS}\left(S_{\infty}\right)$ be its normal subgroup of all permutations which move only finitely many points. By [3], the outer automorphism group of $S_{\infty} / \mathrm{FS}\left(S_{\infty}\right)$ is infinite cyclic. The description of outer automorphisms given in [3] easily shows that if an automorphism $\Phi$ of the Calkin algebra sends the atomic masa to itself, then the restriction of $\Phi$ to the group of all unitaries that send the atomic masa to itself is implemented by a unitary of the Calkin algebra.

The following fact is a major way in which automorphisms of $\mathcal{C}(H)$ differ from automorphisms of $\mathcal{P}(\mathbb{N}) /$ Fin.

Proposition 7.8. An automorphism $\Phi$ of $\mathcal{C}(H)$ is inner if and only if there exists an infinite-dimensional subspace $H_{0}$ of $H$ such that $\Phi \uparrow \mathcal{C}\left(H_{0}\right)$ : $\mathcal{C}\left(H_{0}\right) \rightarrow \mathcal{C}(H)$ is equal to Ad $u$ for a unitary $u: H_{0} \rightarrow H_{1} \subseteq H$.

Proof. Fix $u$ such that $\Phi(b)=u b u^{*}$ for $b \in \mathcal{C}\left(H_{0}\right)$. Fix $v \in \mathcal{C}(H)$ so that $v v^{*}=\pi\left(\operatorname{proj}_{H_{0}}\right)$ and $v^{*} v=I$. Then

$$
\Phi(a)=\Phi\left(v^{*}\right) \Phi\left(v a v^{*}\right) \Phi(v)=\Phi\left(v^{*}\right) u v a v^{*} u^{*} \Phi(v) .
$$

For $w=\Phi\left(v^{*}\right) u v$, we then have $\Phi(a)=w a w^{*}$.

In particular, an automorphism is trivial if and only if it is somewhere trivial. This is not true for automorphisms of $\mathcal{P}(\mathbb{N}) /$ Fin. See, for example, the second part of [51].

7.1. An outer automorphism from the Continuum Hypothesis. Under $\mathrm{CH}$, we might expect to be able to easily modify the proof that $\mathcal{P}(\mathbb{N}) /$ Fin has outer automorphisms to obtain an outer automorphism of $\mathcal{C}(H)$. That is, we would build up an automorphism on separable subalgebras of $\mathcal{C}(H)$, diagonalizing so that it avoids each inner automorphism. However, it turns out that this construction faces serious difficulties at limit stages. Nevertheless, the result still holds. 
Theorem 7.9 (Phillips-Weaver, 2006 [46]). CH implies that the Calkin algebra has outer automorphisms.

The original proof used the approach above, but required very deep $\mathrm{C}^{*}$ algebra machinery to handle limit stages. We instead sketch a more elementary proof, given in [23]. In addition, by an observation of Stefan Geschke, this proof can be easily modified to require not $\mathrm{CH}$ but only $2^{\aleph_{0}}<2^{\aleph_{1}}$ and $\mathfrak{d}=\aleph_{1}$.

Proof of Theorem 7.9. To begin with, fix a basis $\left(e_{n}\right)$. For any partition of $\mathbb{N}$ into finite intervals $\mathbb{N}=\bigcup_{n} J_{n}$, let $E_{n}=\overline{\operatorname{span}}\left\{e_{i}: i \in J_{n}\right\}$ and $\mathcal{D}[\vec{J}]$ be the algebra of all operators that have each $E_{n}$ as an invariant subspace. We write $\vec{J}^{\text {even }}=\left(J_{2 n} \oplus J_{2 n+1}\right)_{n}$ and $\vec{J}^{\text {odd }}=\left(J_{2 n+1} \oplus J_{2 n+2}\right)_{n}$.

Lemma 7.10. Suppose $u$ is a unitary and $\alpha_{n} \in \mathbb{C},\left|\alpha_{n}\right|=1$ for all $n$. Then if $v=\sum_{n} \alpha_{n} P_{J_{n}} u, \operatorname{Ad} u$ and $\operatorname{Ad} v$ agree on $\mathcal{D}[\vec{J}]$.

Proof. Without a loss of generality $u=I$. Note that $a \in \mathcal{D}[\vec{J}]$ if and only if $a=\sum_{n} P_{J_{n}} a P_{J_{n}}$. Thus for $a \in \mathcal{D}[\vec{J}]$,

$$
v a v^{*}=\sum_{n} \alpha_{n} P_{J_{n}} P_{J_{n}} a \overline{\alpha_{n}} P_{J_{n}}=\sum_{n} P_{J_{n}} a P_{J_{n}}=a .
$$

For partitions $\vec{J}, \vec{K}$ of $\mathbb{N}$ into finite intervals we say $\vec{J} \ll \vec{K}$ if for all $m$ there is some $n$ such that $J_{m} \subseteq K_{n} \cup K_{n+1}$.

Lemma 7.11. The ordering $\ll i s ~ \sigma$-directed and cofinally equivalent to $\left(\mathbb{N}^{\mathbb{N}}, \leq^{*}\right)$.

Proof. See $[23, \S 3.1]$.

We write $\mathcal{D} \mathcal{D}[\vec{J}]=\mathcal{D}\left[\vec{J}^{\text {even }}\right] \cup \mathcal{D}\left[\vec{J}^{\text {odd }}\right]$.

Definition 7.12. A family $\mathcal{F}$ of pairs $(\vec{J}, u)$ is a coherent family of unitaries if

(1) $\mathcal{F}_{0}=\{\vec{J}:(\vec{J}, u) \in \mathcal{F}$ for some $u\}$ is $\ll$-cofinal and

(2) For $\vec{J} \ll \vec{K}$ in $\mathcal{F}_{0}$, Ad $u_{\vec{J}}$ and $\operatorname{Ad} u_{\vec{K}}$ agree on $\mathcal{D D}[\vec{J}]$.

The following key lemma is not entirely trivial because not every $a \in \mathcal{B}(H)$ is in $\mathcal{D}[\vec{J}]$ for some $\vec{J}$.

Lemma 7.13. If $\mathcal{F}$ is a coherent family of unitaries then there is the unique automorphism $\Phi_{\mathcal{F}}$ of $\mathcal{C}(H)$ such that $\Phi_{\mathcal{F}}(\pi(a))=\pi\left(u a u^{*}\right)$ for all $(\vec{J}, u) \in \mathcal{F}$ and all $a \in \mathcal{D}[\vec{J}]$.

Proof. The main point is that any $a \in \mathcal{B}(H)$ can be decomposed as $a=$ $a_{0}+a_{1}+c$ so that $a_{0}$ and $a_{1}$ are in $\mathcal{D D}[\vec{E}]$ for some $\vec{E}$ and $c$ is compact ([23, Lemma 1.2]). See [23, Lemma 1.3] for details. 
A coherent family of unitaries is trivial if there is $u_{0} \in \mathcal{B}(H)$ such that $\operatorname{Ad} u_{0}$ and $\operatorname{Ad} u$ agree on $\mathcal{D}[\vec{J}]$ for all $(\vec{J}, u) \in \mathcal{F}$. The automorphism $\Phi_{\mathcal{F}}$ is inner if and only if $\mathcal{F}$ is trivial.

Now by $\mathrm{CH}$ construct $\ll$-increasing and cofinal sequence of partitions $J^{\xi}$ $\left(\xi<\omega_{1}\right)$ and diagonal unitaries $\alpha^{\xi} \in(\mathcal{U}(1))^{\mathbb{N}} \subset \ell^{\infty} \subset \mathcal{B}(H)$ such that for $\xi<\eta$ such that for $\xi<\eta$, Ad $\alpha^{\xi}$ and $\operatorname{Ad} \alpha^{\eta}$ agree on $\mathcal{D}\left[\vec{J}^{\xi}\right]$. This can be done with Lemma 7.10 and some work, and it can be done in such a way that there are $\aleph_{1}$ choices to be made in the construction. We thus obtain $2^{\aleph_{1}}$ different coherent families of unitaries that give $2^{\aleph_{1}}$ different automorphisms of $\mathcal{C}(H)$. Since there are only $2^{\aleph_{0}}$ unitaries in $\mathcal{C}(H)$, some of these automorphisms must be outer.

Note, however, that this construction still does not answer Question 7.6, since the outer automorphisms constructed are locally given by unitaries. The same is true of the automorphisms constructed in Phillips-Weaver's original proof.

7.2. Todorcevic's Axiom implies all automorphisms are inner. The following remarkable axiom was extracted by Todorcevic as one of the key 'Ramseyan' consequences of the Proper Forcing Axiom. See [54] and [42] for the broader context.

Todorcevic's Axiom, TA [53]. Assume $(V, E)$ is a graph such that $E=$ $\bigcup_{n=0}^{\infty} A_{n} \times B_{n}$ for some subsets $A_{n}, B_{n}$ of $V$. Then one of the following applies.

(1) $(V, E)$ has an uncountable clique: $Y \subseteq X$ such that any two vertices in $Y$ are connected by an edge, or

(2) $(V, E)$ is countably chromatic: there is a partition $V=\bigcup_{n=0}^{\infty} X_{n}$ so that no edge connects two vertices in the same $X_{n}$.

Theorem 7.14 (Farah, 2007 [23]). Todorcevic's Axiom implies all automorphisms of $\mathcal{C}(H)$ are inner.

Proof. Fix an automorphism $\Phi$. The proof has two components.

(1) TA implies that the restriction of $\Phi$ to $\mathcal{D}[\vec{J}]$ is implemented by a unitary for every $\vec{J}$.

(2) TA implies that every coherent family of unitaries is trivial.

The proof of (1) is a bit more complicated than the proof of (2), and both can be found in [23].

Assertion (2) is false under $\mathrm{CH}$ (by the proof of Theorem 7.9). On the other hand, we don't know whether (1) is provable without any additional assumption (cf. Theorem 7.7). 


\section{REFERENCES}

1. C. Akemann and N. Weaver, Consistency of a counterexample to Naimark's problem, Proc. Natl. Acad. Sci. USA 101 (2004), no. 20, 7522-7525.

2., $\mathcal{B}(H)$ has a pure state that is not multiplicative on any MASA, Proc. Natl. Acad. Sci. USA 105 (2008), no. 14, 5313-5314.

3. J. L. Alperin, J. Covington, and D. Macpherson, Automorphisms of quotients of symmetric groups, Ordered groups and infinite permutation groups, Math. Appl., vol. 354, Kluwer Acad. Publ., Dordrecht, 1996, pp. 231-247.

4. Joel Anderson, A conjecture concerning the pure states of $B(H)$ and a related theorem, Topics in modern operator theory (Timişoara/Herculane, 1980), Operator Theory: Adv. Appl., vol. 2, pp. 27-43.

5. __ A maximal abelian subalgebra of the Calkin algebra with the extension property, Math. Scand. 42 (1978), no. 1, 101-110.

6 . Extensions, restrictions, and representations of states on $C^{*}$-algebras, Trans. Amer. Math. Soc. 249 (1979), no. 2, 303-329.

7. $\_$Extreme points in sets of positive linear maps on $\mathcal{B}(\mathcal{H})$, J. Funct. Anal. 31 (1979), no. 2, 195-217.

8. William Arveson, An invitation to $C^{*}$-algebras, Springer-Verlag, New York, 1976, Graduate Texts in Mathematics, No. 39.

9. __ A short course on spectral theory, Graduate Texts in Mathematics, vol. 209, Springer-Verlag, New York, 2002.

10. I. Ben Yaacov, A. Berenstein, C.W. Henson, and A. Usvyatsov, Model theory for metric structures, Model Theory with Applications to Algebra and Analysis, Vol. II (Z. Chatzidakis et al., eds.), London Math. Soc. Lecture Notes Series, no. 350, Cambridge University Press, 2008, pp. 315-427.

11. Tristan Bice, Greatest lower bounds of collections of projections in the Calkin algebra, preprint, Kobe University, 2009.

12. B. Blackadar, Operator algebras, Encyclopaedia of Mathematical Sciences, vol. 122, Springer-Verlag, Berlin, 2006, Theory of $C^{*}$-algebras and von Neumann algebras, Operator Algebras and Non-commutative Geometry, III.

13. A. Blass, Cardinal invariants of the continuum, Handbook of Set Theory (M. Foreman and A. Kanamori, eds.), 1999, to appear.

14. L.G. Brown, R.G. Douglas, and P.A. Fillmore, Extensions of $C^{*}$-algebras and $K$ homology, Annals of Math. 105 (1977), 265-324.

15. P.G. Casazza and J.C. Tremain, The Kadison-Singer problem in mathematics and engineering, Proc. Natl. Acad. Sci. USA 103 (2006), no. 7, 2032-2039.

16. John B. Conway, A course in functional analysis, second ed., Graduate Texts in Mathematics, vol. 96, Springer-Verlag, New York, 1990.

17. M. J. Crabb, A new prime $C^{*}$-algebra that is not primitive, J. Funct. Anal. 236 (2006), no. $2,630-633$.

18. Kenneth R. Davidson, $C^{*}$-algebras by example, Fields Institute Monographs, vol. 6, American Mathematical Society, Providence, RI, 1996.

19. G. A. Elliott and A. S. Toms, Regularity properties in the classification program for separable amenable $C^{*}$-algebras, Bull. Amer. Math. Soc. (N.S.) 45 (2008), no. 2, 229245.

20. I. Farah, How many Boolean algebras $\mathcal{P}(\mathbb{N}) / I$ are there?, Illinois J. Math. 46 (2003), 999-1033.

21. _ Rigidity conjectures, Logic Colloquium 2000, Lect. Notes Log., vol. 19, Assoc. Symbol. Logic, Urbana, IL, 2005, pp. 252-271.

22. — A twist of projections in the Calkin algebra, preprint, available at http://www.math.yorku.ca/ ifarah, 2006. 
23. $\ldots$, All automorphisms of the Calkin algebra are inner, preprint, arXiv:0705.3085,

24. The relative commutant of separable $C^{*}$-algebras of real rank zero, Jour. Funct. Analysis 256 (2009), 3841-3846.

25. , Graphs and CCR algebras, Indiana Univ. Math. Journal (to appear).

26. I. Farah, B. Hart, and D. Sherman, Model theory of operator algebras I: Stability, preprint, http://arxiv.org/abs/0908.2790, 2009.

27. __ Model theory of operator algebras II: Model theory, preprint, arXiv:1004.0741, 2010.

28. I. Farah and T. Katsura, Nonseparable UHF algebras I: Dixmier's problem, Adv. Math. (to appear), arXiv:0906.1401v1.

29. I. Farah, N.C. Phillips, and J. Steprāns, The commutant of $L(H)$ in its ultrapower may or may not be trivial, Math. Annalen (to appear), http://arxiv.org/abs/0808.3763v2.

30. L. Ge and D. Hadwin, Ultraproducts of $C^{*}$-algebras, Recent advances in operator theory and related topics (Szeged, 1999), Oper. Theory Adv. Appl., vol. 127, Birkhäuser, Basel, 2001, pp. 305-326.

31. J. Glimm, Type I $C^{*}$-algebras, Ann. of Math. (2) 73 (1961), 572-612.

32. J. G. Glimm and R. V. Kadison, Unitary operators in $C^{*}$-algebras, Pacific J. Math. 10 (1960), 547-556.

33. J.G. Glimm, On a certain class of operator algebras, Trans. Amer. Math. Soc. 95 (1960), 318-340.

34. D. Hadwin, Maximal nests in the Calkin algebra, Proc. Amer. Math. Soc. 126 (1998), 1109-1113.

35. L.A. Harrington, A.S. Kechris, and A. Louveau, A Glimm-Effros dichotomy for Borel equivalence relations, Journal of the Amer. Math. Soc. 4 (1990), 903-927.

36. B. E. Johnson and S. K. Parrott, Operators commuting with a von Neumann algebra modulo the set of compact operators, J. Functional Analysis 11 (1972), 39-61.

37. V.F.R. Jones, Von Neumann algebras, 2003, lecture notes, http://math.berkeley.edu/ vfr/VonNeumann.pdf.

38. R.V. Kadison and I.M. Singer, Extensions of pure states, Amer. J. Math. 81 (1959), 383-400.

39. T. Katsura, Non-separable AF-algebras, Operator Algebras: The Abel Symposium 2004, Abel Symp., vol. 1, Springer, Berlin, 2006, pp. 165-173.

40. K. Kunen, An introduction to independence proofs, North-Holland, 1980.

41. Н. Лузин, О частях натурального ряда, Изв. АН СССР, серия мат. 11, №5 (1947), $714-722$.

42. J. Tatch Moore, The proper forcing axiom, Proceedings of the ICM 2010, 2010.

43. E. Odell and Th. Schlumprecht, The distortion problem, Acta Mathematica 173 (1994), 259-281.

44. G.K. Pedersen, $C^{*}$-algebras and their automorphism groups, London Mathematical Society Monographs, vol. 14, Academic Press Inc. [Harcourt Brace Jovanovich Publishers], London, 1979.

45. _ Analysis now, Graduate Texts in Mathematics, vol. 118, Springer-Verlag, New York, 1989.

46. N.C. Phillips and N. Weaver, The Calkin algebra has outer automorphisms, Duke Math. Journal 139 (2007), 185-202.

47. G. A. Reid, On the Calkin representations, Proc. London Math. Soc. (3) 23 (1971), $547-564$.

48. M. Rørdam, Classification of nuclear $C^{*}$-algebras., Encyclopaedia of Mathematical Sciences, vol. 126, Springer-Verlag, Berlin, 2002, Operator Algebras and Noncommutative Geometry, 7.

49. Shôichirô Sakai, $C^{*}$-algebras and $W^{*}$-algebras, Classics in Mathematics, SpringerVerlag, Berlin, 1998, Reprint of the 1971 edition. 
50. S. Shelah, Proper forcing, Lecture Notes in Mathematics 940, Springer, 1982.

51. S. Shelah and J. Steprāns, Non-trivial homeomorphisms of $\beta N \backslash N$ without the continuum hypothesis, Fundamenta Mathematicae 132 (1989), 135-141.

52. _ Masas in the Calkin algebra without the continuum hypothesis, Preprint, 2009.

53. S. Todorcevic, Partition problems in topology, Contemporary mathematics, vol. 84, American Mathematical Society, Providence, Rhode Island, 1989.

54. Combinatorial dichotomies in set theory, Bull. Symb. Logic (to appear).

55. N. Weaver, Mathematical quantization, Studies in Advanced Mathematics, Chapman \& Hall/CRC, Boca Raton, FL, 2001.

56. - The kadison-Singer problem in discrepancy theory, preprint, arXiv:math.CO/0209078, 2002.

57. _ A prime $C^{*}$-algebra that is not primitive, J. Funct. Anal. 203 (2003).

58. _ Set theory and $C^{*}$-algebras, Bull. Symb. Logic 13 (2007), 1-20.

59. E. Wofsey, $P(\omega) /$ fin and projections in the Calkin algebra, Proc. Amer. Math. Soc. 136 (2008), no. 2, 719-726.

60. B. Zamora Aviles, The structure of order ideals and gaps in the Calkin algebra, Ph.D. thesis, York University, 2009.

61. R. J. Zimmer, Essential results of functional analysis, Chicago Lectures in Mathematics, University of Chicago Press, Chicago, IL, 1990.

Dept. of Mathematics and Statistics, York University, 4700 Keele Street, Toronto, Canada M3J 1P3

Matematicki Institut, Kneza Mihaila 34, 11000 Beograd, Serbia

E-mail address: ifarah@mathstat.yorku.ca

$U R L:$ http://www.mathstat.yorku.ca/ ifarah

Department of Mathematics, Washington University in Saint Louis, Saint LOUIS, MO 63130

Current address: Department of Mathematics, Harvard University, One Oxford Street, Cambridge MA 02138 